PNL-7291

UC-402

\title{
Uncertainty in North America Wet Deposition Isopleth Maps: Effect of Site Selection and Valid Sample Criteria
}

J. C. Simpson

A. R. Olsen

April 1990

Prepared for the

U.S. Environmental Protection Agency under a Related Services Agreement with the U.S. Department of Energy

Contract DE-AC06-76RLO 1830

Pacific Northwest Laboratory Operated for the U.S. Department of Energy by Battelle Memorial Institute 


\title{
DISCLAIMER
}

This report was prepared as an account of work sponsored by an agency of the United States Government. Neither the United States Government nor any agency thereof, nor Battelle Memorial Institute, nor any of their employees, makes any warranty, expressed or implied, or assumes any legal liability or responsibility for the accuracy, completeness, or usefulness of any information, apparatus, product, or process disclosed, or represents that its use would not infringe privately owned rights. Reference herein to any specific commercial product, process, or service by trade name, trademark, manufacturer, or otherwise, does not necessarily constitute or imply its endorsement, recommendation, or favoring by the United States Government of any agency thereof, or Battelle Memorial Institute. The views and opinions of authors expressed herein do not necessarily state or reflect those of the United States Government or any agency thereof.

\author{
PACIFIC NORTHWEST LABORATORY \\ operated by \\ BATTELLE MEMORIAL INSTITUTE \\ for the \\ UNITED STATES DEPARTMENT OF ENERGY \\ under Contract DE-AC06-76RLO 1830
}

Printed in the United States of America

Available to DOE and DOE contractors from the

Office of Scientific and Technical Information, P.O. Box 62, Oak Ridge, TN 37831; prices available from (615) 576-8401. FTS 626-8401.

Available to the public from the National Technical Information Service, U.S. Deparment of Commerce, 5285 Port Royal Rd., Springfield, VA 22161.

NTIS Price Codes, Microfiche A01

Printed Copy

\begin{tabular}{cr}
\hline Price Code & Page Range \\
\hline A02 & $1-10$ \\
A03 & $11-50$ \\
A04 & $51-75$ \\
A05 & $76-100$ \\
A06 & $101-125$ \\
A07 & $126-150$ \\
A08 & $151-175$ \\
A09 & $176-200$ \\
A10 & $201-225$ \\
A11 & $226-250$ \\
A12 & $251-275$ \\
A13 & $276-300$ \\
A14 & $301-325$
\end{tabular}

\begin{tabular}{cc}
\hline Price Code & Page Range \\
\hline A15 & $326-350$ \\
A16 & $351-375$ \\
A17 & $376-400$ \\
A18 & $401-425$ \\
A19 & $426-450$ \\
A20 & $451-475$ \\
A21 & $476-500$ \\
A22 & $501-525$ \\
A23 & $526-550$ \\
A24 & $551-575$ \\
A25 & $576-600$ \\
A99 & $601-U p$
\end{tabular}


PNL -7291

UC -402

UNCERTAINTY IN NORTH AMERICA

WET DEPOSITION ISOPLETH MAPS:

EFFECT OF SITE SELECTION AND VALID

SAMPLE CRITERIA

J. C. Simpson

A. R. 01sen

April 1990

Prepared for the

U.S. Environmental Protection Agency under a Related Services Agreement with the U.S. Department of Energy

Contract DE-AC06-76RL0 1830

Pacific Northwest Laboratory

Richland, Washington 99352 
-

.

.

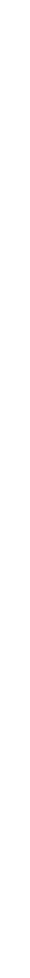




\section{ABSTRACT}

This report considers several issues related to the preparation of isopleth maps for the display of spatial patterns of wet deposition. The valid sample criteria and data completeness rating used in the data summarization process are described. The data interpolation technique, kriging, is presented and it's derivation in terms of generalized least squares regression is given. Four different annual summaries for $\mathrm{pH}$, sulfate concentration, and sulfate deposition in 1986 are prepared using either the Unified Deposition Database Committee (UDDC) definition of valid sample criteria or a relaxed valid sample criteria and the UDDC data completeness rating or a relaxed data completeness rating. The kriged estimates for the different annual summaries and the differences between these estimates are contoured. The effects of relaxing the valid sample criteria and data completeness rating are discussed. Conclusions are drawn about network operation, network design and the uncertainty of contour maps. It is recommended that in the case where the objective is contour maps to show regional patterns, the emphas is in most regions needs to be on the number of valid samples per site and the regional representativeness of the sites. 
*

-

,

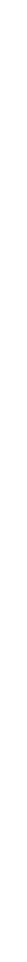

. 


\section{CONTENTS}

ABSTRACT ..........................

1.0 INTRODUCTION . . . . . . . . . . . . . . 1.1

2.0 DESCRIPTION OF WET DEPOSITION DATA SETS . . . . . . 2.1

2.1 WET DEPOSITION DATA SOURCES . . . . . . . . 2.1

2.2 DATA SUMMARIZATION PROCESS . . . . . . . . 2.3

2.3 SITE SELECTION PROCESS .................. 2.5

2.4 DEFINITION OF ALTERNATIVE DATA SETS USED IN STUDY $\ldots . .2 .9$

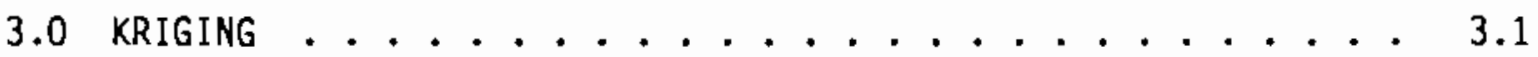

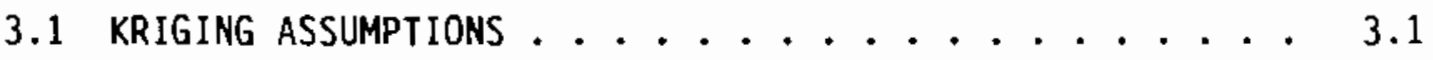

3.2 SEMI-VARIOGRAM ESTIMATION . . . . . . . . . . . 3.2

3.3 KRIGING ESTIMATOR ..................... 3.5

3.4 GENERALIZED LEAST SQUARES (GLS) REGRESSION $\ldots \ldots \ldots$

3.5 DERIVATION OF THE GLS ESTIMATOR . . . . . . . . . 3.7

3.6 WITHIN-SITE VARIATION ....................... 3.10

3.7 ADVANTAGES OF GLS APPROACH . . . . . . . . 3.11

4.0 VARIOGRAM ESTIMATES $\ldots \ldots \ldots \ldots . \ldots \ldots$

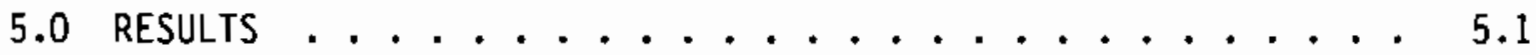

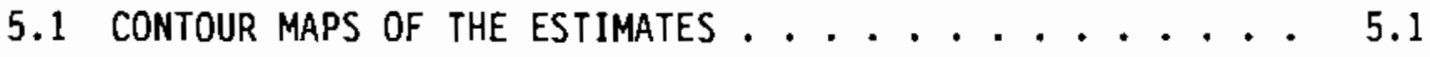

5.2 CONTOUR MAPS OF THE DIFFERENCES . . . . . . . 5.9

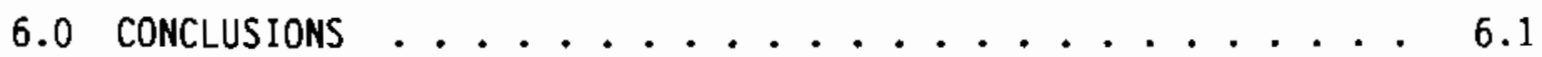

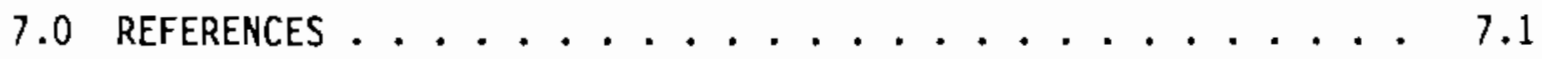

APPENDIX A - COMPARISON OF 1985 PH CONTOUR MAPS . . . . . . A.1 


\section{FIGURES}

2.1 Location of Sites in the Relaxed Valid Sample Criteria and Relaxed Data Completeness Rating Subset ....... 2.10

3.1 Semi-Variogram Models where the Sill and Range are One for All the Models Except the Power Models where " $b$ " is Set to One and the Semi-Variogram is Truncated

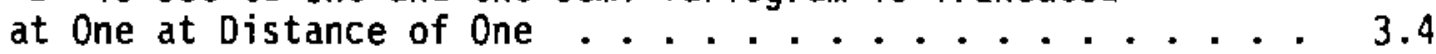

4.1 Semi-Variogram for Each of the Subsets of pH, Sulfate Concentration and Sulfate Distributions ........ 4.3

5.1 Contours of pH Estimates Using the UDDC Valid Sample Criteria/UDDC Data Completeness Rating Subset . . . . . 5.2

5.2 Contours of Sulfate Concentrations (mg/l) Estimates Using the UDDC Valid Sample Criteria/UDDC Data Completeness Rating Subset ......... 5.3

5.3 Contours of Sulfate Depositions ( $\mathrm{g} / \mathrm{sq} \mathrm{m})$ Estimates Using the UDDC Valid Sample Criteria/UDDC Data Completeness Rating Subset ............. 5.4

5.4 Contours of $\mathrm{pH}$ Estimates of the Four Subsets Using the UDDC or Relaxed Valid Sample Criteria (UVSC or RVSC) and the UDDC or Relaxed Data Completeness Rating (UDCR or RDCR) . . . . . . 5.6

5.5 Contours of Sulfate Concentration (mg/1) Estimates of the Four Subsets Using the UDDC or Relaxed Valid Sample Criteria (UVSC or RVSC) and the UDDC or Relaxed Data Completeness Rating (UDCR or RDCR) . . . . 5.7

5.6 Contours of Sulfate Deposition ( $\mathrm{g} / \mathrm{sq} \mathrm{m})$ Estimates of the Four Subsets Using the UDDC or Relaxed Valid Sample Criteria (UVSC or RVSC) and the UDDC or Relaxed Data Completeness Rating (UDCR or RDCR) ........ 5.8

5.7 Comparison of Sulfate Concentration and Deposition Estimates With and Without Parsons, West Virginia Site Present ......... 5.10

5.8 Differences in pH Estimates for Selected Pairs of Subsets . . 5.11

5.9 Differences in Sulfate Concentration Estimates for Selected Pairs of Subsets .......... 5.12 
5.10 Differences in Sulfate Deposition Estimates for

Selected Pairs of Subsets ............. 5.13

5.11 Extent and Magnitude of Effect the Parsons, West Virginia Site has on the Sulfate Concentration

and Deposition Estimates............. 5.18 


\section{TABLES}

2.1 Definition of Data Completeness Measures . . . . . . . 2.8

2.2 Data Completeness Level Criteria for Annual Summaries . . . 2.9

2.3 The Number of Sites for Each of the Four Sets of $\mathrm{pH}$ and Sulfate Sunmaries ............ 2.11

2.4 The Number of Sites where $\mathrm{pH}$ Values Changed by the UDDC Versus Relaxed Valid Sample Criteria and the Magnitudes of Those Changes .......... 2.12

2.5 The Number of Sites where Sulfate Values Changed by the UDOC Versus Relaxed Valid Sample Criteria and the Magnitudes of Those Changes .......... 2.13

2.6 The 16 Sites with the Largest Changes in their $\mathrm{pH}$ and/or Sulfate Concentrations as a Result of Using the Relaxed Versus UDDC Valid Sample Criteria . . . . 2.14

2.7 The Samples Within the 16 Sites which Did Not Meet the UDDC Valid Sample Criteria ....... 2.16 


\subsection{INTRODUCTION}

Junge (1963) operated the first precipitation chemistry network in the United States and reported the data by using isopleth maps of ion concentrations. Since then a number of regional and national isopleth maps of precipitation chemistry data have been published: Semonin (1981), Cowling (1982), Calvert et al. (1983), Munger and Eisenreich (1983), and Barrie and Hales (1984). The researchers determined the location of the contours on these maps either by subjectively using expert opinion to hand draw them or simple weighting schemes such as inverse distance squared.

More recently several researchers have used the geostatistical technique termed kriging to estimate the spatial surface and then display the surface using isopleth maps. Eynon and Switzer (1983) and Finkelstein (1984) published isopleth maps based on two alternative kriging approaches. Since then a number of authors have used kriging in the production of maps: Seilkop and Finkelstein (1987), Barchet (1987), Wampler and 0lsen (1987), Guertin et al. (1988), Gibson et al. (1989) and Venkatram (1988). Bilonick (1985) and Le and Petkau (1988) applied spatial time series models to precipitation chemistry data in North America.

The National Atmospheric Deposition Program (NADP) and the Acid Deposition System (ADS) regularly publish United States and North American isopleth maps of annual precipitation chemistry. NADP coordinates the NADP/NTN network and uses the network data to produce annual maps starting with 1983 data. NADP (1987) is an example of their publications. The ADS combines the NADP/NTN network data with data from several other North American precipitation chemistry monitoring networks to produce annual isopleth maps for North America. See 01sen and Watson (1984), 01sen and Slavich (1985), 01sen and Slavich (1986), Sweeney and 0lsen (1987), Sweeney and 01 sen (1989), and 01sen (1989). NADP uses a constrained distancesquared weighting function to estimate the surface while ADS uses kriging to estimate the surface. A more detailed explanation comparing the approaches is given in an informal report by 0isen (1988), which is reproduced as Appendix A. 
In 1988, researchers concerned with wet deposition data organized two spatial analysis workshops. The first workshop on regional analys is of wet deposition for effects research was held June 1988 in Corvallis, Oregon. Vong et al. (1989) sumarize the workshop discussion. The workshop focused on issues connected with estimating wet deposition at non-monitored sites and did not explicitly consider presentation of regional wet deposition surfaces as isopleth maps. One conclusion of the workshop is that kriging is currently a preferred technique for interpolation to non-monitored sites. The second workshop focused explicitly on presentation of isopleth maps and was held October 1988 in Champaign, Illinois. The workshop organizers prepared standard data sets for North America and invited six different organizations to use the standard data sets to produce isopleth maps. Methods used by the organizations included hand drawn by an expert, inverse distance squared, Cressman's objective weighting, other objective analys is schemes, and severat kriging alternatives. No workshop report is available. Our conclusions from the workshop are that regardless of the interpolation technique the broad overall regional features of the surface are very similar across techniques but local features do differ when different techniques are used.

The production of an isopleth map involves: 1) selection of sites to be used in surface estimation, 2) calculation of an annual sumnary value at a monitored site, 3) selection of a surface estimation technique, 4) selection on how to display the estimated surface, and 5) production of a final document quality display. Decisions made for each of these can affect the final display and how it is perceived. When the surface is displayed using isopleth maps, alternative choices can result in different maps. Even if agreement on these issues could be reached, the wet deposition data has measurement error associated with the sampling and laboratory analysis process. Hence an isopleth map has uncertainty associated with the location of its isopleth lines due to measurement error and to the uncertainty associated with possible alternative production decisions.

Isopleth maps are a common method of sumnarizing and displaying the spatial pattern for wet deposition over North America. The process includes 
determining annual summaries at wet deposition monitoring sites, selecting sites with representative data, estimating a spatial surface by interpolating the selected sites to a regular grid, and then displaying the surface using an isopleth map. It is generally recognized that the location of the isopleth lines can be affected by the procedures used at each stage of the process. In fact, isopleth maps for the same annual time period and ion species constructed by different organizations have been constructed, for example, the pH map for 1985 given in the NAPAP Interim Assessment report (Barchet 1987) and the $\mathrm{pH}$ map for 1985 in the NADP/NTN Annual Data Summary report (NADP 1987). The maps do differ and the question arises as to why. A natural follow-up question is can an agreement be reached on what the "correct" process is to go from sample data collected at sites to the "correct" isopleth map.

An isopleth map is a display of an estimated surface. Data at monitored sites used in the surface estimation process are only estimates of wet deposition at the monitored site. Hence, the location of the isopleth lines would still have uncertainty associated with them even if organizations would agree to use the same data, same sites and same surface estimation methodology. The uncertainty of the data at the site and the uncertainty associated with estimating wet deposition at non-monitoring locations as part of the surface estimation methodology both contribute to the uncertainty in the location of the isopleth lines. A natural question is how to determine (estimate) the uncertainty on the location of the isopleth lines.

This report investigates the impact on estimated surfaces due to the use of alternative procedures for estimating wet deposition at individual monitoring sites and for selecting representative monitoring sites. The impact of alternative surface estimation methodologies is not explicitly investigated. 


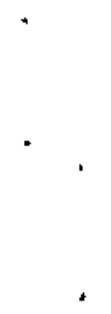




\subsection{DESCRIPTION OF WET DEPOSITION DATA SETS}

The purpose of this section is: 1) to describe wet deposition monitoring data sources, data summarization processes, and site selection processes that have been used by organizations who produce isopleth maps for wet deposition ion species and 2) define alternative data sets used in this report. No attempt is made to include all procedures that have been used. The discussion is focussed on the processes used to define the alternative data sets used later in the report.

\subsection{WET DEPOSITION DATA SOURCES}

Various federal, state, and local governmental agencies and private industry organizations support networks of sites for the collection and chemical analysis of precipitation samples, i.e., for wet deposition monitoring. Determining which networks of sites to include is a primary decision in a study of wet deposition spatial patterns. Issues typically considered in the selection process are: 1) objectives and spatial coverage of the network, 2) quality assurance associated with the data, 3) compatibility of network monitoring, laboratory, and data validation protocols, 4) availability of data, and 5) special requirements/restrictions by spatial pattern study organization. The first three issues are associated with the representativeness and compatibility of the data. The latter two issues are typically operational issues rather than representativeness issues. The selection of different networks to be included in spatial pattern studies for the same year can potentially be a major source of differences between isopleth maps prepared by two organizations. Interpretation or comparison of isopleth maps requires an understanding and assessment of the network selection criteria used.

The wet deposition data used for this study are from six regional or national networks that contribute data to the Acid Deposition System (ADS) (Watson and 01sen 1984). The networks are the Multi-State Atmospheric Pollution and Power Production Study initiated precipitation chemistry network (MAP3S/PCN), the National Atmospheric Deposition Program/National 
Trends Network (NADP/NTN), the Utility Acid Precipitation Study Program (UAPSP), the Canadian Acid Precipitation Monitoring Network (CAPMON), the Acidic Precipitation in Ontario Study daily (APIOS-D) and cumulative (APIOS-C) networks. Sweeney and 01 sen (1989) give additional descriptions of the networks. Criteria used in selecting these networks are: 1) the network provides regional or national coverage at regionally representative sites, 2) each network has an implemented quality assurance program, 3) the network chemical analys is laboratories participate in an inter-laboratory comparison program, and 4) the data are readily available in a common format from ADS.

The decision to use these networks does impact the spatial pattern displayed by an isopleth map. Site selection protocols for the networks restricts location of sites within urban areas and minimizes impact of local point source emissions. Although not an explicit site selection protocol, almost all sites are located away from mountainous regions. Consequently, isopleth maps based on the networks may not reflect variations in the spatial surface related to local point sources effects, urban influences, or elevation effects. Any spatial pattern derived will at best reflect the broad regional or national structure of wet deposition. The impact of this decision on isopleth maps will not be explicitly studied in the report.

The networks used in the study each have a chemical analysis laboratory that performs sample analyses and a data management function that checks the reasonableness of the sample analys is results using information available from the analysis and from the sample field notes. That is, each network receives data from a laboratory which has been subjected to internal laboratory checks and to sampling protocol checks. The checks result in supporting comments and data flags being attached to the samples. The ADS data base incorporates all of the comnents, codes and flags. A quality assurance program is used by each of the networks to ensure that their protocols are implemented and their laboratories are in control. In addition, all of the laboratories participate in interlaboratory comparison studies. 


\subsection{DATA SUMMARIZATION PROCESS}

Calculation of an annual summary from sample data collected at a site requires criteria for assessing whether a sample data value is valid or invalid for the purposes of the study. These criteria are called valid sample criteria. Even with careful attention, some samples collected must be declared invalid because of the violation of some aspect of a network's protocol that affects the sample's representativeness of the precipitation chemistry. In some cases, such as severe contamination by debris, the sample is clearly not representative of the precipitation chemistry. In other cases, such as bulk sampling or non-protocol sample period, whether the sample is representative of the precipitation chemistry during the period is not as clear. Currently, a sample's representativeness and a criteria for a valid sample have not been generally developed and accepted. valid sample criteria that do exist are based on the best professional judgments of wet deposition monitoring researchers.

To study the impact of different valid sample criteria, hence indirectly sample representativeness, two alternative valid sample criteria have been defined for the study. One alternative uses the valid sample criteria developed by the Unified Deposition Database Committee (UDDC) (0lsen et al. 1990). The other alternative relaxes the UDDC valid sample criteria by removing two UDDC valid sample criteria. This alternative is termed the relaxed valid sample criteria.

The UDDC valid sample criteria have been designed to incorporate each network's comments, codes and flags into the decision process of determining whether an individual wet deposition sample result is to be included or excluded from a summary. The discussion on screening for valid samples is stated in terms of the ADS data base common record format (Watson and 01sen 1984) with some reference to network specific codes as necessary for clarification.

All networks include note codes which are informational in nature. Some codes denote reasons why sample results are not available or reported. other codes describe conditions present in the field, and during sample 
transit and sample receipt. Unless explicitly stated elsewhere, these note codes are not used in determining whether a sample is valid. The basic premise is that each network has screened individual sample results for possible contamination. If a sample result passes the network's screening, it is assumed that possible sample contamination indicated by field or lab comments did not materially affect the sample ion species concentrations.

A set of valid sample criteria has been designed for each network. Each sample associated with a sampling period is screened to determine whether the sample meets specific criteria. The screening criteria use the informational comments and codes provided by each network. The criteria are:

- All sampling periods for which it is known that no precipitation occurred are considered valid sample periods. This applies mainly to weekly, monthly and 28-day sampling protocols. For event and daily sampling protocols the absence of a sample record for a day implies that no precipitation occurred.

- The wet deposition sample must be a wet-only sample. All samples identified as bulk, partially bulk or undefined are invalid.

- Wet deposition samples that have insufficient precipitation to complete a chemical analysis for a specific ion species are invalid for that specific ion species. Event/daily samples are most likely to have this occur.

- An individual ion species concentration accompanied by a comment code designating the measurement to be "suspect" or "invalid" is declared an invalid sample. Deletion of the ion species concentration by the network for the same reason has the same result.

- The actual sampling period for a wet deposition sample must be close to the network's protocol sampling period. Specifically, the following conditions lead to an invalid sample:

For NADP/NTN, actual sample period less than 6 days or greater than 8 days. This includes all NADP/NTN samples coded "LD" with measured precipitation.

For APIOS-C, actual sample period less than 21 days or greater than 35 days.

For UAPSP, actual sample period greater than 1 day. 
The restriction to wet-only samples used for the UDDC valid sample criteria may be too stringent and their exclusion may result in a bias. For the networks used in the study, the sample chemistry is checked for internal consistency and for consistency with historical data from the site. Hence severe contamination due to an exposed bucket will cause other data flags to be set. Large differences between wet-only and bulk sample chemistry may not be common in networks designed with wet-only sampling protocols. Typically, the sampler fails at some time during the sampling period, violating the protocol. Exclusion of bulk samples can cause a bias in annual precipitation weighted mean concentrations, especially if an ion species has a seasonal pattern at a site. It may be more appropriate to include a bulk sample as the best estimate of concentration rather than exclude the sample and in effect use the annual precipitation weighted mean concentration as the estimate for the sample. No detailed study has been completed to study this question.

The UDDC valid sample criteria also restricts the actual sample period length of a sample to be within a few days of the network's protocol sampling period. For example, NADP/NTN samples must be 6,7 , or 8 days in length. The reason for the restriction is to maintain consistency within the data. Again however, no objective study has been completed to show the effect of departure from a daily, weekly, or four-weekly sampling period. The criteria may be overly restrictive and introduce a bias as stated above for bulk samples.

\subsection{SITE SELECTION PROCESS}

Calculation of an annual summary based on valid data at a site does not imply that the annual summary represents the chemistry of precipitation that occurred at the site during the year. This may be due to the sampling not being completed continuously at the site, to incomplete collection of individual precipitation events (low collection efficiency), to chemical changes occurring in the sample during or after sample collection, or to precipitation associated with invalid samples having concentrations different than that associated with valid samples (e.g., seasonal effects). 
The study defines and uses two alternative site selection criteria for assessing the completeness, i.e., representativeness, of the annual summary. One is the UDDC data completeness criteria which incorporates both an annual and a quarterly criteria. The other, called the relaxed data completeness criteria, is a relaxed UDDC criteria which eliminates the UDDC quarterly criteria and weakens the UDDC annual criteria.

The Unified Deposition Database Committee defined five quantitative data completeness measures and assigned annual and quarterly thresholds to each of the five measures in constructing their UDDC data completeness criteria. The following questions concerning data completeness and temporal representativeness motivated the measures: for what portion of the summary period is the occurrence and amount of precipitation known; what portion of the precipitation volume collected is associated with valid deposition samples; what percent of the time and what percent of the samples collected are associated with valid samples; and what is the ratio of the wet deposition sample volume to the precipitation measured by a standard gage?

Data completeness measures are based on the assumption that the entire season or year consists of sample periods that account for every day of the summary period. It is normal for a site to have incomplete information for some precipitation events during a summary period, to deviate from established collection protocols due to circumstances outside the operator's control, or to collect samples that are subsequently eliminated during the network's data screening process. Therefore, it is necessary to establish criteria for determining when sufficient valid wet deposition data are present to calculate a meaningful seasonal or annual sumary for a site. Data completeness measures are designed to quantify the amount of information upon which a data sumnary is based and enable criteria to be established that indicate the quality of the summary. Five data completeness measures are proposed: percent precipitation coverage length, percent total precipitation, percent valid sample length, percent of samples with measured precipitation that are valid and percent collection efficiency. A sixth measure, percent sea salt correction is applied to 
sulfate summaries for sites within $100 \mathrm{~km}$ of a coast. Definitions for the data completeness measures are given in Table 2.1.

The data completeness measures are the basis for assigning a data completeness level ( 1 to 4 ) to each seasonal and annual summary. The criteria for the data completeness levels are given in Table 2.2. A summary with data completeness level 1 has the best information, or the highest level of data completeness. The least confidence is given to a summary with data completeness level 3. Level 4 summaries fail level 3 criteria. They are viewed as not providing a representative summary for the period. In order for a data summary to be assigned a specific level, all criteria listed for the level must be met. The most favorable level attained is assigned to the summary. A summary that does not meet one or more of the criteria for level 3 is assigned as level 4.

The collection efficiency data completeness level criteria for a seasonal summary is relaxed somewhat for Canadian winter sumnaries compared to other seasons due to the generally poorer collector performance for snow sampling. If the criteria for other seasons is applied to winter months when a large percentage of the precipitation in Canada is in the form of snow, then only a few locations meet even the level 3 criterion. It is believed that a lower percentage could be accepted for winter because the problems are primarily due to undercatch of snow. An under-collected snow sample may reasonably represent the concentration but not the deposition.

The data completeness level for an annual sumbary is based on annual criteria as well as criteria for the four quarters, January-March, AprilJune, July-September, and October-December, which comprise the year. The addition of quarterly criteria to the annual criteria is to ensure that adequate data from each quarter is present in the annual summary. Because the emphasis is on insuring adequate data for an annual summary, some quarterly criteria are relaxed from the seasonal criteria (see Table 2.2). 
TABLE 2.1. Definition of Data Completeness Measures

Data

Completeness

Measure

Definition

$\because P C L \quad$ Percent precipitation coverage length is the percent of the summary period for which information on whether or not precipitation occurred is available. If precipitation is known to have occurred during a particular sampling period but no measurement of the amount is available, then no knowledge of precipitation is assumed. This measure can be less than $100 \%$ because the site started (stopped) operation after (before) the beginning (end) of the summary period or because equipment or operator problems caused the site to be shut down for a portion of the summary period.

\&TP Percent total precipitation is the percent of the total precipitation depth measured that is associated with valid samples collected during the summary period.

\&VSL

Percent valid sample length is the percent of the days during the sumnary period for which valid samples are obtained. Note that sample periods with no precipitation are considered valid samples.

FVSMP Percent valid samples with measured precipitation is the percent of all wet deposition samples during the summary period that are valid samples.

$\because$ COLEFF Percent collection efficiency is the ratio of the wet deposition sample volume (converted to a depth) to the total precipitation depth as measured by a collocated rain gage. only valid samples with both a collocated standard rain gage and sample volume measurement available are used.

\%SEASALT Percent sea salt correction is the percent of the average sulfate concentration that is estimated to be due to sea salt, using sodium or magnesium as tracers of sea salt. 
TABLE 2.2. Data Completeness Level Criteria for Annual Summaries

\begin{tabular}{|c|c|c|c|}
\hline Data Completeness Measure & $\begin{array}{l}\text { Annual Dat } \\
1\end{array}$ & $\begin{array}{c}\text { Comp leteness } \\
2\end{array}$ & $\begin{array}{r}\text { Level } \\
3 \\
\end{array}$ \\
\hline $\begin{array}{l}\text { } \mathrm{PCL} \\
\text { Annual and } \\
\text { each quarter }\end{array}$ & $\begin{array}{l}\geq 95 \% \\
\geq 75 \%\end{array}$ & $\begin{array}{l}\geq 90 \% \\
\geq 60 \%\end{array}$ & $\begin{array}{l}\geq 90 \% \\
\geq 50 \%\end{array}$ \\
\hline $\begin{array}{l}\text { \%TP, \%VSL, \%VSMP } \\
\text { Annual and } \\
\text { each quarter }\end{array}$ & $\begin{array}{l}\geq 80 \% \\
\geq 70 \%\end{array}$ & $\begin{array}{l}\geq 70 \% \\
\geq 60 \%\end{array}$ & $\geq 60 \%$ \\
\hline $\begin{array}{l}\text { \%OOLEFF } \\
\text { Annual and } \\
\text { for winter and } \\
\text { spring, summer, autumn }\end{array}$ & $\begin{array}{l}>80 \%(70 \%)^{*} \\
\geq 80 \%(50 \%)^{*} \\
\geq 80 \%\end{array}$ & $\begin{array}{l}\geq 60 \%(40 \%)^{*} \\
\geq 60 \%(40 \%)^{*} \\
\geq 60 \%\end{array}$ & $\begin{array}{l}\geq 50 \%(30 \%)^{*} \\
\geq 50 \%(30 \%)^{*} \\
\geq 50 \%\end{array}$ \\
\hline$\because$ SEASALT & $\leq 25 \%$ & $\leq 50 \%$ & $\leq 75 \%$ \\
\hline
\end{tabular}

* The bracketed value applies to Canadian sites.

The relaxed data completeness criteria applies only to the annual $\% \mathrm{PCL}$ $(\geq 90 \%)$ and $\%$ TP $(\geq 60 \%)$ data completeness measures. All quarterly criteria are dropped. Sites meeting the relaxed data completeness criteria but not the UDDC criteria, almost exclusively fail the quarterly criteria. The quarterly criteria are intended to ensure that all quarters of the year are represented in an annual summary. However, the criteria may be overly restrictive. The relaxed criteria result in almost all sites that monitored continuously during a year being included in the study.

\subsection{DEFINITION OF ALTERNATIVE DATA SETS USED IN STUDY}

The UDOC valid sample criteria (UVSC) versus relaxed valid sample criteria (RVSC) and the UDDC data completeness rating (UDCR) versus relaxed data completeness rating (RDCR) create four subsets of sites (UVSC/UDCR, UVSC/RDCR, RVSC/UDCR and RVSC/RDCR) for both the $\mathrm{pH}$ and sulfate observations. The locations of the sites in the RVSC/RDCR subset are shown in Figure 2.1. The estimation technique used in this study (kriging) requires that the phenomenon be stationary in the region being investigated. For $\mathrm{pH}$ and wet deposition of sulfate, this stationarity requirement is not 


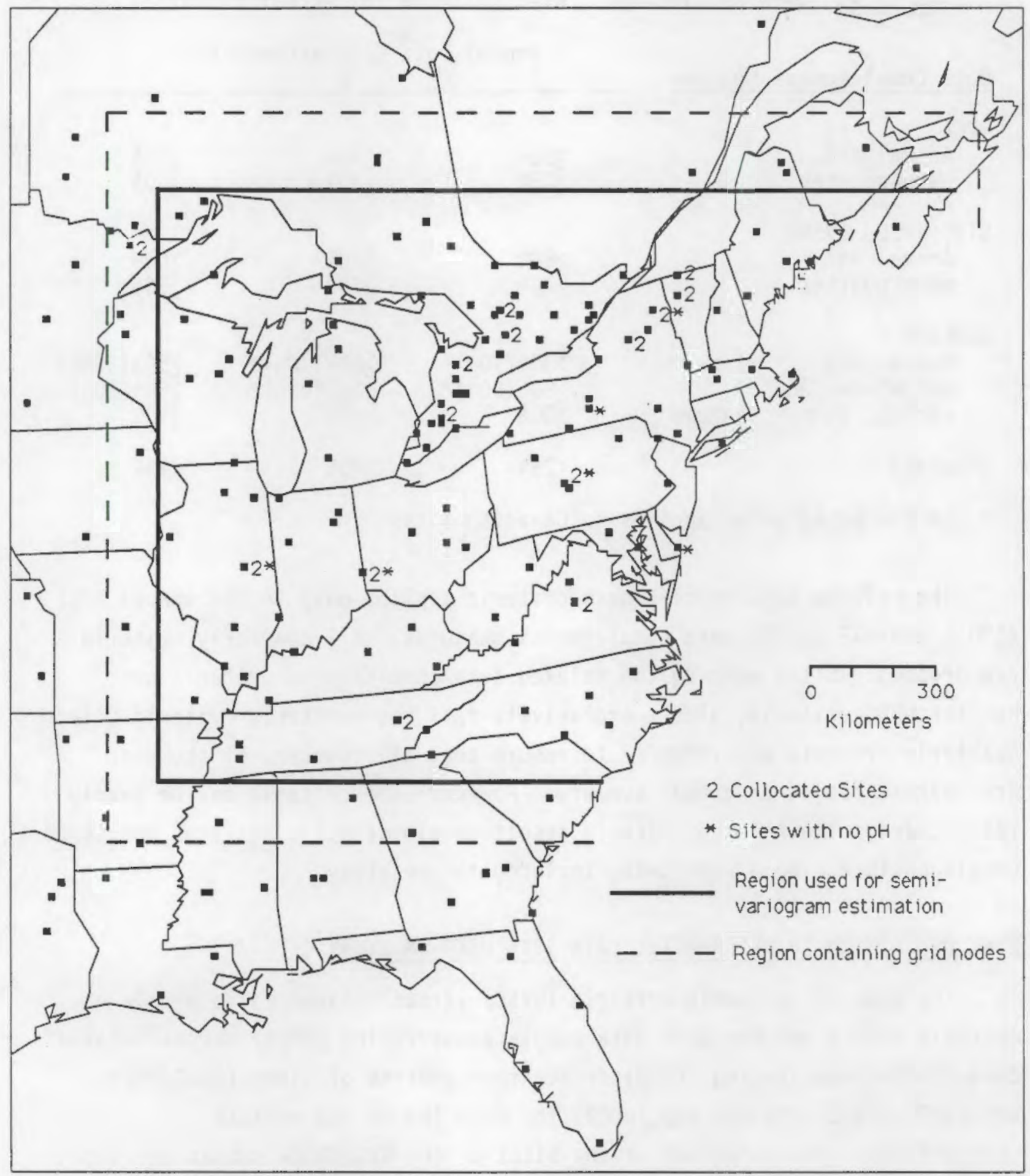

FIGURE 2.1. Location of Sites in the Relaxed Valid Sample Criteria and Relaxed Data Completeness Rating Subset 
met when considering the entire United States and southern Canada as one region (Vong et. al. 1989). Therefore this study is restricted to southern Canada and the eastern United States (less the southern most states). As seen in Figure 2.1, the $\mathrm{pH}$ observations are at the same sites as the sulfate observations with the exception of the six sites without $\mathrm{pH}$ observations. The number of sites in each of the subsets are shown in Table 2.3. Of the 194 sites, in the region shown in Figure 2.1, that have $\mathrm{pH}$ and sulfate summaries in 1986, 185 sites (95.4\%) have annual $\mathrm{pH}$ summaries and 191 sites (98.5\%) have annual sulfate summaries which meet the minimal requirements (RVSC/RDRC). When the most stringent requirements (UVSC/UDCR) are used, only 113 sites (58.2\%) have annual pH summaries and 125 sites (64.4\%) have annual sulfate summaries which meet these requirements. The difference between the UDCR and RDCR is solely in the number of sites in the subsets. The primary difference between the UVSC and RVSC subsets is that at approximately one third of the sites the summaries change in value. Additionally, the RVSC subsets have a few more sites, since more samples are included in the annual summary causing the data completeness rating to improve.

The number of sites whose observations changed and the magnitude of those changes are shown in Tables 2.4 and 2.5, for $\mathrm{pH}$ and sulfate respectively. As seen in Tables 2.4 and 2.5, the majority of the changes in the observations are relatively small. The 16 sites, within the region where estimates are calculated (see Figure 2.1), with the largest changes

TABLE 2.3. The Number of Sites for Each of the Four Sets of pH and Sulfate Summaries

\begin{tabular}{lll}
$\mathrm{pH}$ & UVSC & RVSC \\
RDCR & 184 & 185 \\
UDCR & 113 & 122 \\
$\mathrm{SO}_{4}$ & $\underline{\text { UVSC }}$ & RVSC \\
\hline RDCR & 190 & 191 \\
UDCR & 125 & 133
\end{tabular}


TABLE 2.4. The Number of Sites Where $\mathrm{pH}$ Values Changed by the UDDC Versus Relaxed Valid Sample Criteria and the Magnitudes of Those Changes

\begin{tabular}{crr} 
(RVSC - UVSC) & UDCR & RDCR \\
\cline { 2 - 3 }$(-0.127,-0.100)$ & 1 & 2 \\
$(-0.100,-0.050)$ & 3 & 3 \\
$(-0.050,-0.025)$ & 1 & 3 \\
$(-0.025,-0.005)$ & 11 & 13 \\
$(-0.005,0.000)$ & 10 & 13 \\
no change & 80 & 114 \\
$(0.000,0.005)$ & 9 & 14 \\
$(0.005,0.025)$ & 5 & 7 \\
$(0.025,0.050)$ & 2 & 5 \\
$0.050,0.096)$ & 0 & 2
\end{tabular}

in their observations are shown in Table 2.6. As seen in this table, most of the large changes in $\mathrm{pH}$ and sulfate are at the same sites.

Table 2.7 shows the samples from the 16 sites shown in Table 2.6 which did not meet the UDDC valid sample criteria. There are two causes for these samples to be rejected, either the collector is open during the collection period when it is not raining (bulk) or the sample collection period violates the networks protocol by being either too long or too short. The precipitation weighted average of $\mathrm{pH}$ and sulfate concentration are given for most of the samples, as data validation procedures of the networks did not indicate anything unusual about the chemistry of these samples. As seen in Table 2.7, three of the sites each have samples with extremely high sulfate concentration (greater that $50 \mathrm{mg} / 1$ ). Although there is little precipitation associated with these samples, even when precipitation weighted averages are calculated, these samples have a significant effect on the average. These unusual samples also have extremely small collection efficiencies (percent of predicted sample volume, from the rain gage, that is contained in the actual sample). For a number of other sites, the samples that did not meet the UDDC valid sample criteria all occur contiguously in time (the NADP samples represent one week while for the APIOS-C network one sample represents a four week period). For sites that 
TABLE 2.5. The Number of Sites Where Sulfate Values Changed by the UDDC Versus Relaxed Valid Sample

Criteria and the Magnitudes of Those Changes

$\mathrm{SO}_{4}$ Concentration $(\mathrm{mg} / \mathrm{l})$

\begin{tabular}{|c|c|c|}
\hline (RVSC-UVSC) & $\underline{\text { UDCR }}$ & $\underline{\mathrm{RDCR}}$ \\
\hline 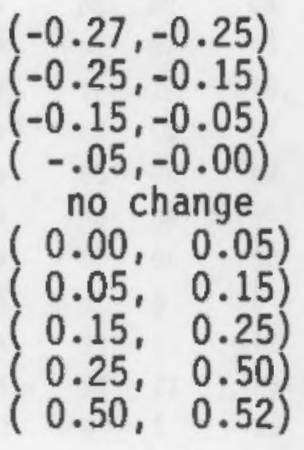 & $\begin{array}{r}0 \\
0 \\
2 \\
14 \\
89 \\
11 \\
9 \\
4 \\
3 \\
1\end{array}$ & $\begin{array}{r}1 \\
2 \\
3 \\
19 \\
120 \\
16 \\
11 \\
6 \\
4 \\
1\end{array}$ \\
\hline \multicolumn{3}{|c|}{$\mathrm{SO}_{4}$ Deposition $\left(\mathrm{g} / \mathrm{m}^{2}\right)$} \\
\hline (RVSC-UVSC) & $\underline{\text { UDCR }}$ & $\underline{\mathrm{ROCR}}$ \\
\hline 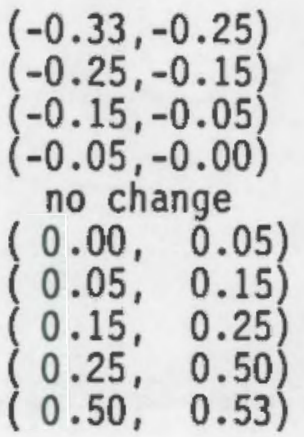 & $\begin{array}{r}0 \\
0 \\
2 \\
14 \\
149 \\
89 \\
11 \\
8 \\
3 \\
5 \\
1\end{array}$ & $\begin{array}{r}1 \\
2 \\
2 \\
20 \\
120 \\
16 \\
10\end{array}$ \\
\hline
\end{tabular}

have a seasonal trend, the loss of a month or more of data can significantly raise or lower the annual average.

A fifth subset of observations for the sulfate observations is investigated. This set consisted of the UVSC/UDCR subset with the extreme value at Parsons, West Virginia (ADS ID 075a) removed. This subset is used to demonstrate the range and magnitude of the effect that one unusual site can have. 
TABLE 2.6. The Sixteen Sites with the Largest Changes in Their pH and/or Sulfate Concentrations as a Result of Using the Relaxed Versus UDDC Valid Sample Criteria

ADS

Site

ID

ต32a

943b

647a

$973 a$

977a

163a

168 a

$187 a$

188 .

192a

288a

241a

25\%a

278a

426a

495a

\begin{tabular}{|c|c|c|}
\hline Network & Sitenane & $\begin{array}{l}\text { Precip } \\
\text { (ca) }\end{array}$ \\
\hline NADP & Kellogg, Michigan & 125 \\
\hline NADP & Thiteface, New York & 117 \\
\hline NADP & Jasper, New York & 83 \\
\hline NADP & Horton Station, Virginia & 96 \\
\hline NADP & Spooner, Wisconsin & 79 \\
\hline NADP & Caribou, Maine & 88 \\
\hline NADP & Hunt ington, New York & 109 \\
\hline APIOS-C & Uxbridge, Ontario & 94 \\
\hline APIOS-C & Vilberforce, Ontario & 99 \\
\hline APIOS-C & Saith's Falls, Ontario & 97 \\
\hline APIOS-C & Lac Le Croix, Ontario & 58 \\
\hline UAPSP & Gaylord, Michigan & 65 \\
\hline NADP & Big Uoadows, Virginia & 165 \\
\hline NADP & Cadwe II, Massachuetts & 113 \\
\hline NADP & Vincennes, Indiana & 115 \\
\hline APIOS-C & Woosonee, Ontario & 62 \\
\hline
\end{tabular}

* Total Number of

Precip Sanples $\underline{\text { W }} \underline{\mathrm{RV}}$ W RV $\begin{array}{llllll}\text { RD } & \text { RD } & 60 & 89 & 29 & 36\end{array}$ $\begin{array}{llllll}\text { RD UD } & 77 & 84 & 46 & 44\end{array}$ $\begin{array}{llllll}R D & \text { UD } & 75 & 81 & 41 & 42\end{array}$ $\begin{array}{llllll}R D & R D & 63 & 75 & 32 & 34\end{array}$ $\begin{array}{llllll}\text { UD UD } & 92 & 94 & 36 & 38\end{array}$ $\begin{array}{lllll}\text { UD UD } & 99 & 169 & 48 & 50\end{array}$ $\begin{array}{llllll}\text { RD UD } & 75 & 83 & 39 & 46\end{array}$ \begin{tabular}{l|lllll} 
RD & RD & 65 & 73 & 8 & 10
\end{tabular} $\begin{array}{llllll}R D & \text { UD } & 75 & 94 & 16 & 12\end{array}$ $\begin{array}{llllll}R D & R D & 88 & 160 & 11 & 12\end{array}$ $\begin{array}{lllllll}\text { RD } & \text { RD } & 68 & 89 & 7 & 9\end{array}$ $\begin{array}{llllll}\text { UO } & \text { UD } & 89 & 97 & 73 & 75\end{array}$ $\begin{array}{lllllll}\text { RD } & \text { RD } & 62 & 69 & 23 & 27\end{array}$ $\begin{array}{lllllll}R D & \text { NO } & 82 & 96 & 33 & 37\end{array}$ $\begin{array}{llllll}R D & \text { UD } & 83 & 94 & 31 & 38\end{array}$

\begin{tabular}{|c|c|c|}
\hline \multicolumn{3}{|c|}{$\mathrm{pH}$} \\
\hline W & RV & Difference \\
\hline 4.372 & 4.400 & +0.028 \\
\hline 4.373 & 4.369 & ஏ. \\
\hline 4.261 & 4.295 & -9.056 \\
\hline 4.383 & 4.418 & +0.635 \\
\hline 4.898 & 4.878 & -8.026 \\
\hline 4.617 & 4.694 & -8.613 \\
\hline 4.351 & 4.325 & -6.626 \\
\hline 4.251 & 4.282 & +8.831 \\
\hline 4.299 & 4.342 & +6.043 \\
\hline 4.323 & 4.289 & -0.934 \\
\hline 5. 116 & 4.889 & -0.127 \\
\hline 4.511 & 4.454 & -6.657 \\
\hline 4.438 & 4.410 & -6.628 \\
\hline 4.397 & 4.310 & +6.603 \\
\hline 4.388 & 4.289 & -6.697 \\
\hline 4.852 & 4.947 & +6.695 \\
\hline
\end{tabular}

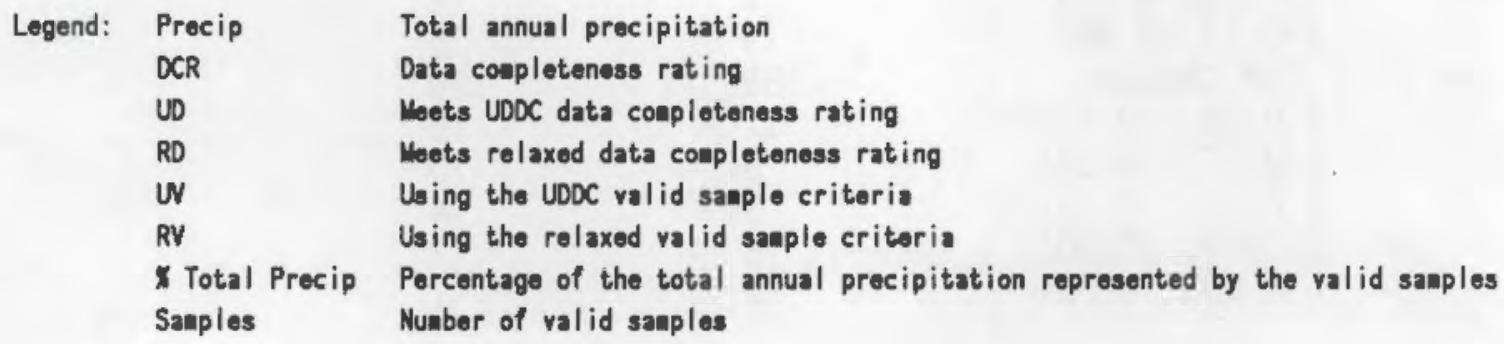




\section{TABLE 2.6 (Continued).}

\begin{tabular}{|c|c|c|c|c|c|c|c|c|c|c|c|c|}
\hline \multirow{3}{*}{$\begin{array}{l}\text { ADS } \\
\text { Site } \\
\text { ID } \\
\end{array}$} & & & \multirow{2}{*}{\multicolumn{2}{|c|}{$\begin{array}{l}\text { X Total } \\
\text { Precip } \\
\end{array}$}} & \multirow{2}{*}{\multicolumn{2}{|c|}{$\begin{array}{c}\text { Number of } \\
\text { Sapples }\end{array}$}} & \multicolumn{6}{|c|}{ SUFATE } \\
\hline & \multicolumn{2}{|c|}{ DCR } & & & & & \multicolumn{3}{|c|}{ Concentration (ag/I) } & \multicolumn{3}{|c|}{ Deposition $\left(\mathrm{g} / \mathrm{m}^{2}\right)$} \\
\hline & $\underline{\mathrm{w}}$ & RV & $\underline{U Y}$ & RV & $\underline{\mathbf{U V}}$ & $\underline{\text { RV }}$ & W & RV & Difference & UN & RV & Difference \\
\hline $632 a$ & RD & RD & 66 & 89 & 20 & 36 & 2.743 & 2.475 & -6.268 & 3.416 & 3.982 & -6.334 \\
\hline g43b & RD & RD & 77 & B4 & 48 & 44 & 1.967 & 2.184 & +0.217 & 2.398 & 2.581 & +6.253 \\
\hline $047 a$ & RD & UD & 75 & 81 & 41 & 42 & 2.564 & 3.921 & +9.458 & 2.139 & 2.521 & +0.382 \\
\hline $973 a$ & RD & RD & 63 & 75 & 32 & 34 & 2.463 & 2.238 & $-\$ .225$ & 2.376 & 2.154 & -0.216 \\
\hline 677a & UD & UD & 92 & 94 & 38 & 38 & 1.436 & 1.758 & +0.322 & 1.134 & 1.388 & +0.254 \\
\hline $163 a$ & UD & UD & 99 & 160 & 48 & 50 & 1.319 & 1.838 & +0.517 & 1.158 & 1.689 & +6.453 \\
\hline $168 a$ & RD & UD & 75 & 83 & 39 & 45 & 2.679 & 2.190 & +0.111 & 2. 281 & 2.382 & +6.121 \\
\hline $187 a$ & RD & RD & 85 & 73 & 8 & 10 & 3.573 & 3.341 & -0.232 & 3.348 & 3.131 & -0.217 \\
\hline $188 a$ & UD & UD & 85 & 84 & 16 & 12 & 2.816 & 2.818 & +6.602 & 2.794 & 2.796 & +0.602 \\
\hline $192 a$ & RD & RD & 62 & 69 & 23 & 27 & 2.955 & 3.114 & +6.150 & 2.864 & 3.018 & +0.154 \\
\hline $288 a$ & RD & RD & 78 & 160 & 9 & 11 & 1.348 & 1.383 & +0.634 & 6.789 & B. 809 & +6.020 \\
\hline $241 a$ & UD & UD & 89 & 97 & 73 & 75 & 1.588 & 1.824 & +0.238 & 1.340 & 1.541 & +0.201 \\
\hline $25 \% a$ & RD & RD & 62 & 69 & 23 & 27 & 1.925 & 2.184 & +0.259 & 2.613 & 2.284 & +6.271 \\
\hline $276 a$ & RD & UD & 87 & 96 & 42 & 44 & 2.078 & 2.215 & +0.137 & 2.352 & 2.508 & +6.156 \\
\hline $426 a$ & RD & UD & 83 & 94 & 31 & 38 & 2.626 & 3.681 & +0.461 & 3.620 & 3.551 & +0.531 \\
\hline $495 a$ & RD & RD & 67 & 83 & 6 & 8 & 1.442 & 1.468 & -6.636 & 0.887 & 1.865 & -6.022 \\
\hline
\end{tabular}

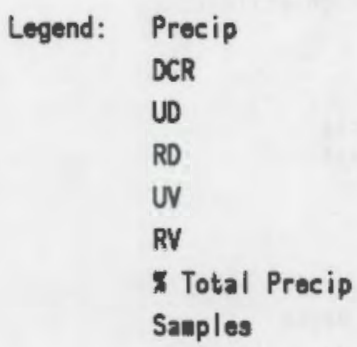

Total annual precipitation Data conpleteness rating Weets UDDC data completeness rating Wots relaxed data completeness rating Using the UDOC valid sample criteria Using the relaxed valid sanple criteria Percentage of the total annual precipitation represented by the valid saaples Nunber of valid sanples 
TABLE 2.7. The Samples Within the 16 Sites (Shown in Table 2.6) Which Did Not Meet the UDDC Valid Sample Criteria

\begin{tabular}{|c|c|c|c|c|c|c|c|}
\hline $\begin{array}{l}\text { ADS } \\
\text { SITE ID } \\
\end{array}$ & $\underline{N}$ & CAUSE & PRECIP & $\begin{array}{l}\text { X TOTAL } \\
\text { PRECIP } \\
\end{array}$ & $\mathrm{pH}$ & $\begin{array}{l}\text { SUFATE } \\
\operatorname{CONC}(\mathrm{g} / 1)\end{array}$ & COMENTS \\
\hline 632a & $\begin{array}{l}1 \\
6\end{array}$ & $\begin{array}{l}\text { period } \\
\text { period }\end{array}$ & $\begin{array}{l}25.6 \\
11.2\end{array}$ & $\begin{array}{l}26.6 \\
9.6\end{array}$ & $\begin{array}{l}4.860 \\
4.334\end{array}$ & $\begin{array}{l}1.450 \\
3.646\end{array}$ & no sanple volune \\
\hline $0.43 b$ & $\begin{array}{l}1 \\
3\end{array}$ & $\begin{array}{l}\text { bulk } \\
\text { bulk }\end{array}$ & $\begin{array}{l}3.5 \\
4.3\end{array}$ & $\begin{array}{l}3.6 \\
3.6\end{array}$ & $\begin{array}{l}4.190 \\
4.566\end{array}$ & $\begin{array}{l}7.796 \\
2.152\end{array}$ & $\begin{array}{l}\text { July } \\
\text { July }\end{array}$ \\
\hline 647a & 1 & bulk & 4.4 & 5.3 & 3.770 & 9.566 & \\
\hline $673 a$ & 2 & period & 12.0 & 12.4 & 4.662 & 1.102 & \\
\hline $677 a$ & $\begin{array}{l}1 \\
1\end{array}$ & $\begin{array}{l}\text { bulk } \\
\text { bulk }\end{array}$ & $\begin{array}{l}6.8 \\
6.3\end{array}$ & $\begin{array}{l}1.0 \\
0.4\end{array}$ & $\begin{array}{l}8.44 \\
3.75\end{array}$ & $\begin{array}{r}1.250 \\
74.650\end{array}$ & $5.8 x$ collection efficiency \\
\hline $163 a$ & 2 & bulk & 6.9 & 1.0 & 4.726 & 51.995 & $\begin{array}{l}11.2 x \text { and } 24.8 \% \text { collection } \\
\text { officiency }\end{array}$ \\
\hline $168 a$ & $\begin{array}{l}4 \\
2 \\
1\end{array}$ & $\begin{array}{l}\text { bulk } \\
\text { bulk } \\
\text { bulk }\end{array}$ & $\begin{array}{l}5.5 \\
3.0 \\
6.1\end{array}$ & $\begin{array}{l}5.2 \\
2.8 \\
6.1\end{array}$ & $\begin{array}{l}4.158 \\
4.182 \\
3.869\end{array}$ & $\begin{array}{l}2.441 \\
4.734\end{array}$ & Fobruary and Wareh \\
\hline $187 a$ & 2 & period & 7.5 & 8.6 & 4.686 & 1.478 & \\
\hline $188 a$ & 2 & bulk & 18.7 & 18.9 & 4.761 & 2.826 & \\
\hline $192 a$ & 1 & bulk & 13.5 & 13.9 & 4.120 & 4.160 & \\
\hline $208 a$ & $\begin{array}{l}1 \\
1\end{array}$ & $\begin{array}{l}\text { period } \\
\text { period }\end{array}$ & $\begin{array}{l}2.7 \\
9.9\end{array}$ & $\begin{array}{l}4.7 \\
16.9\end{array}$ & $\begin{array}{l}5.620 \\
4.570\end{array}$ & $\begin{array}{l}0.860 \\
1.700\end{array}$ & $35.1 \times$ collection efficiency \\
\hline 241a & 2 & period & 6.1 & 7.3 & 4.653 & 4.744 & \\
\hline $25 \% a$ & $\begin{array}{l}2 \\
2\end{array}$ & $\begin{array}{l}\text { bulk } \\
\text { bulk }\end{array}$ & $\begin{array}{l}5.8 \\
2.2\end{array}$ & $\begin{array}{l}5.6 \\
2.1\end{array}$ & $\begin{array}{l}4.542 \\
4.844\end{array}$ & $\begin{array}{r}1.540 \\
11.321\end{array}$ & $\begin{array}{l}\text { March and April } \\
\text { March and April }\end{array}$ \\
\hline $276 a$ & $\begin{array}{l}1 \\
1\end{array}$ & $\begin{array}{l}\text { bulk } \\
\text { bulk }\end{array}$ & $\begin{array}{l}2.5 \\
8.2\end{array}$ & $\begin{array}{l}2.2 \\
7.2\end{array}$ & $\begin{array}{l}6.98 \\
4.23\end{array}$ & $\begin{array}{l}5.824 \\
2.750\end{array}$ & \\
\hline $426 a$ & $\begin{array}{l}6 \\
1\end{array}$ & $\begin{array}{l}\text { bulk } \\
\text { bulk }\end{array}$ & 12.4 & $\begin{array}{l}16.8 \\
6.4\end{array}$ & $\begin{array}{l}4.556 \\
2.686\end{array}$ & $\begin{array}{r}3.996 \\
69.326\end{array}$ & $\begin{array}{l}\text { Fobruary and Warch } \\
2.9 x \text { collection officiency }\end{array}$ \\
\hline 495a & $\begin{array}{l}1 \\
1 \\
1\end{array}$ & $\begin{array}{l}\text { period } \\
\text { period } \\
\text { period }\end{array}$ & $\begin{array}{l}2.2 \\
2.2 \\
7.8\end{array}$ & $\begin{array}{l}3.6 \\
3.5 \\
12.7\end{array}$ & $\begin{array}{l}4.640 \\
7.120 \\
6.210\end{array}$ & $\begin{array}{l}1.106 \\
1.366\end{array}$ & $\begin{array}{l}\text { November } \\
\text { Decenber, 2.6x collection efficiency }\end{array}$ \\
\hline
\end{tabular}




\subsection{KRIGING}

To investigate the effects of using the four different subsets of the observations, the observations are interpolated onto a regular grid using a variation of kriging. Kriging has often been the tool used to make predictions of a spatial phenomenon (e.g. sulfate deposition) at unobserved sites (e.g. a grid node). Kriging's popularity is based on the fact that the estimates it produces are "sensible" and it also produces a variance that is often used in setting confidence intervals about the estimates.

\subsection{KRIGING ASSUMPTIONS}

Let $Z(\underline{x})$ be a realization of a spatial phenomenon. For example, $Z(\underline{x})$ can be the sulfate deposition at a site and $\underline{x}$ is the location of the site in two dimensional space $(\underline{x}=(x, y))$. "Simple" kriging assumes that the increments (difference between the sulfate deposition at two sites), $\left[Z\left(\underline{x}^{\prime}\right)-Z(\underline{x})\right]$, are stationary in the weak sense. That is,

$$
E\left[Z\left(\underline{x}^{\prime}\right)-Z(\underline{x})\right]=0
$$

and

$$
\operatorname{VAR}\left[Z\left(\underline{x}^{\prime}\right)-Z(\underline{x})\right]=2 \gamma(h)
$$

where $\quad h=$ distance between $\underline{x}$ and $\underline{x}^{\prime}$

$$
\gamma(h)=\text { semi-variogram . }
$$

Equation (1) states that the expected difference between the two sites is zero or that the expected sulfate depositions are constant over the region of interest. Equation (2) states that the increment has a variance and this variance is a function (called the semi-variogram) only of the distance between the two sites.

When there is a systematic change (drift or trend) in the spatial phenomenon, then

$$
E[Z(\underline{x})]=m(\underline{x})
$$


where $m(\underline{x})$ is usually modeled as a low order polynomial. Now, using Equation (3),

$$
E\left[Z\left(\underline{x}^{\prime}\right)-Z(\underline{x})\right]=m\left(\underline{x}^{\prime}\right)-m(\underline{x}) \text {. }
$$

and

$$
\operatorname{VAR}\left[Z\left(\underline{x}^{\prime}\right)-Z(\underline{x})\right]=2 \gamma(h)-\left[m\left(\underline{x}^{\prime}\right)-m(\underline{x})\right]^{2} .
$$

Thus, the increments no longer have a constant expected value and the variance of the increments is a function of both the semi-variogram and the drift. Now the variance of the increments cannot be modeled by the semi-variogram alone. The problem at this point is that to be able to estimate the drift, the semi-variogram needs to be known, and to be able to estimate the semi-variogram, the drift needs to be known. Unfortunately, neither are known.

In practice, often the drift is ignored. There are two common justifications given for ignoring the drift. First, the actual estimates derived from kriging only use a subset of the sites near the point being estimated, thus if the drift is "small" in the "neighborhood" of the point being estimated then the drift's effect on the estimates will be negligible. The second justification is that "what's drift to one person is correlation to the next."

\subsection{SEMI-VARIOGRAM ESTIMATION}

As seen in Equation (2), under the assumption that the increments are stationary in the weak sense, the variance of the increments are modeled by the semi-variogram. The semi-variogram model must be of a form so that the variance is non-negative.

Five of the more common semi-variogram models which give non-negative values are:

\section{Power Model}

$$
\gamma(h)=b|h|^{p} \quad \text { for } 0<p<2
$$

(when $p=1$, the semi-variogram is simply a linear model) 
2. Spherical Model

$$
\begin{array}{ll}
\gamma(h)=c\left[\frac{3}{2} \frac{|h|}{r}-\frac{1}{2} \frac{|h|^{3}}{r^{3}}\right] & \text { for }|h| \leq r \\
\gamma(h)=c & \text { for }|h|>r
\end{array}
$$

3. Cubic Model

$$
\begin{array}{ll}
\gamma(h)=c\left[7 \frac{|h|^{2}}{r^{2}}-\frac{35}{4} \frac{|h|^{3}}{r^{3}}+\frac{7}{2} \frac{|h|^{5}}{r^{5}}-\frac{3}{4} \frac{|h|^{7}}{r^{7}}\right] & \text { for }|h| \leq r \\
\gamma(h)=c & \text { for }|h|>r
\end{array}
$$

4. Exponential Model

$$
\gamma(h)=c\left[1-e^{-|h| /(r / 3)}\right]
$$

5. Gaussian Model

$$
\gamma(h)=C\left[\left(1-e^{-|h|^{2} /(r / 2)^{2}}\right]\right.
$$

In the above equations, $r$ equals the range of the semi-variogram and $C$ equals the sill. The range can be thought of as the "zone of influence". If the distance between two sites is less than the range, then the value of one site influences the value of the other site. If the distance between two sites is greater than the range, then the sites are independent. The sill is the bound on the semi-variogram and provides an estimate of the overall variability. The power model does not have a range or a sill. The exponential and gaussian models never reach their sill. Figure 3.1 gives a comparison of these semi-variogram models where the sill and range are one for all the models except the power models where b is set to one and the semi-variogram is truncated at one at distance one. As seen in Figure 3.1 , a wide range of semi-variograms can be modeled using these five models. 


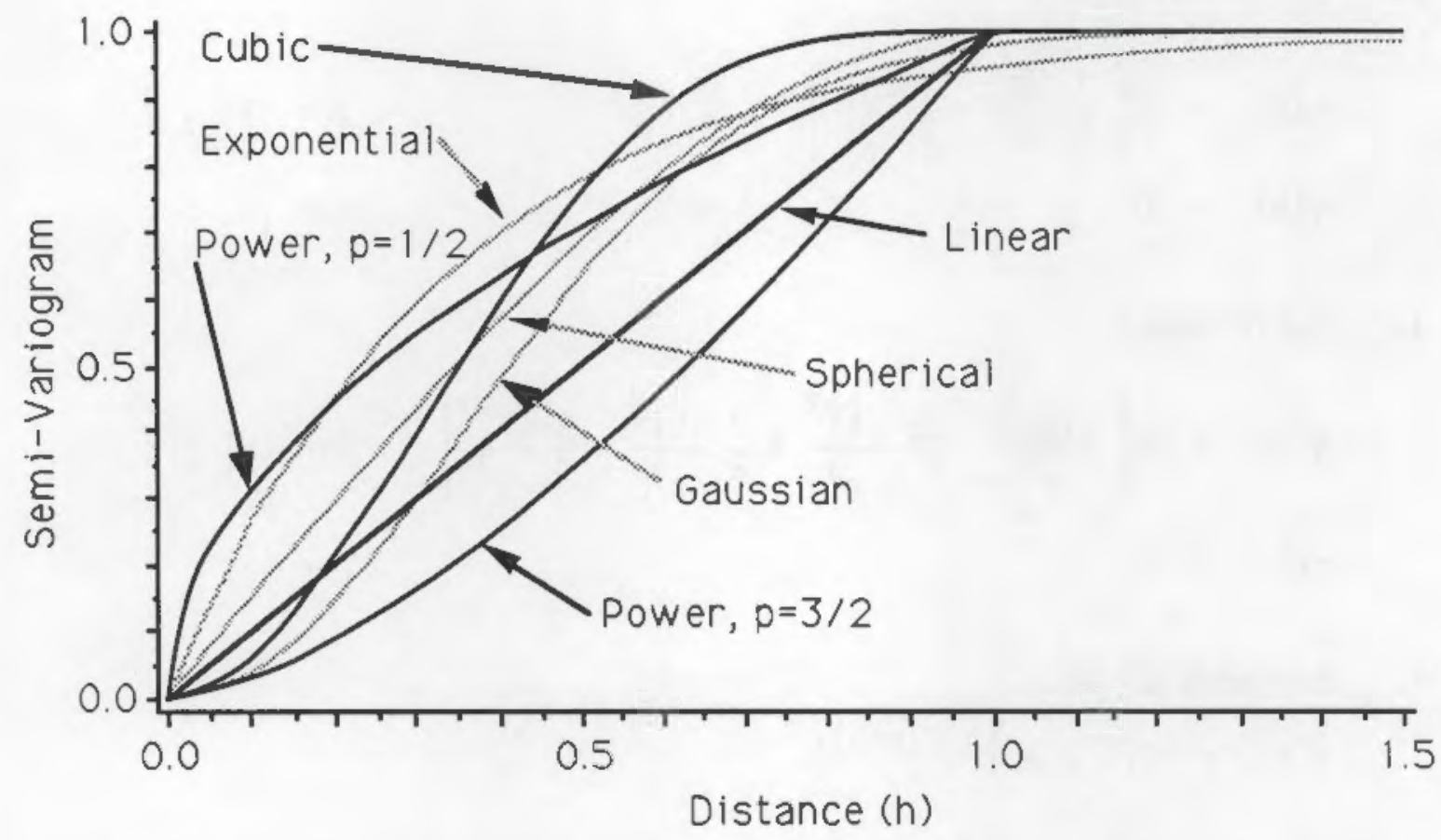

FIGURE 3.1. Semi-Variogram Models Where the Sill and Range are are One for All the Models Except the Power Models Where " $b$ " is Set to One and the Semi-Variogram is Truncated at One at Distance of One

When $h$ is zero, $\gamma(0)$ must also be equal to zero. However, if the semivariogram does not tend to zero for measurements taken at arbitrarily close points, then there is a discontinuity of the semi-variogram at the origin. This discontinuity is called the nugget effect. If there is a nugget effect, the variogram model is adjusted to take it into account. For example, if the model is linear with a nugget of size $c_{0}$ (the intercept) then

$$
\begin{array}{ll}
\gamma(h)=b|h|+C_{D} & \text { for }|h|>0 \\
\gamma(h)=0 & \text { for }|h|=0 .
\end{array}
$$

The semi-variogram is estimated by 


$$
\hat{\boldsymbol{\gamma}}(h)=\frac{1}{2 N(h)} \sum_{i=1}^{N(h)}\left[z\left(\underline{x}_{i}+h\right)-Z\left(\underline{x}_{i}\right)\right]^{2}
$$

where $\quad z\left(\underline{x}_{j}+h\right)-z\left(\underline{x}_{j}\right)=$ difference between a pair of observations which are a distance $h$ apart

$$
\begin{aligned}
N(h)= & \text { number of pairs of points actually taken } \\
& \text { into the sum. }
\end{aligned}
$$

In practice $h$ is a range of distances.

\subsection{KRIGING ESTIMATOR}

The kriging estimator is

$$
\hat{Y}_{0}=\sum_{i=1}^{n} \lambda_{i} z\left(\underline{x}_{i}\right)
$$

where

$$
\begin{aligned}
\hat{y}_{0} & =\text { the kriging estimator at grid node } \underline{x}_{0} \\
\lambda_{i} & =\text { kriging weights } \\
Z\left(\underline{x}_{i}\right) & =\text { the observed sulfate deposition at site } \underline{x}_{i} \\
n & =\text { the number of sites used in the estimator. }
\end{aligned}
$$

The number of sites used in the estimator, $n$, is only a small fraction of the total number of sites (generally between 8 and 16). Only "close" observations are used to reduce the size of the matrices that need to be manipulated. As long as the semi-variogram model has a small nugget as compared to the sill, then the weights decrease rapidly with distance from $\underline{x}_{0}$ and thus observations that are "far" from $\underline{x}_{0}$ have negligible weight.

The "simple" kriging variance is

$$
\sigma_{k}^{2}=2 \sum_{i=1}^{n} \lambda_{i} \gamma\left(\underline{x}_{i}, \underline{x}_{0}\right)-\sum_{i=1}^{n} \sum_{j=1}^{n} \lambda_{i} \lambda_{j} \gamma\left(\underline{x}_{j}, \underline{x}_{j}\right)
$$


where $\quad \boldsymbol{\gamma}\left(\underline{x}_{j}, \underline{x}_{j}\right)=$ semi-variogram, $\boldsymbol{\gamma}(h)$ where $h$ is the distance between $\underline{x}_{i}$ and $\underline{x}_{j}$.

If the semi-variogram has a sill and there is no drift, the kriging variance may be stated in terms of variances and covariances,

$$
\sigma_{k}^{2}=\sum_{i=1}^{n} \sum_{j=1}^{n} \lambda_{i} \lambda_{j} \sigma\left(\underline{x}_{i}, \underline{x}_{j}\right)-2 \sum_{i=1}^{n} \lambda_{i} \sigma\left(\underline{x}_{i}, \underline{x}_{0}\right)+\sigma^{2}
$$

where

$$
\begin{aligned}
\sigma\left(\underline{x}_{j}, \underline{x}_{j}\right) & =\text { covariance between } \underline{x}_{i} \text { and } \underline{x}_{j} \\
& =\sigma^{2}-\gamma\left(\underline{x}_{j}, \underline{x}_{j}\right) \\
\sigma^{2} & =\text { variance of } y_{0}(\text { sill). }
\end{aligned}
$$

\subsection{GENERALIZED LEAST SQUARES (GLS) REGRESSION}

Least squares regression assumes that the errors in the observations are independent. That is, the deviations from a trend surface fit using least squares regression and the actual observations are independent. If it assumed that these errors are not independent, then generalized least squares regression can be used to estimate the trend surface. For example, in kriging the dependence in the errors is assumed to be a function only of the distance between the observations. Because these errors are related, they also form a "surface" that fits on top of the trend surface. Therefore, it makes sense to also estimate the error "surface" and then add it to the trend surface.

\subsection{DERIVATION OF THE GLS ESTIMATOR}

The GLS model is

$$
\underline{Y}=X \underline{\beta}+\underline{\epsilon}
$$

where $\quad \underline{Y}^{\prime}=\left(Y_{1}, Y_{2}, \ldots, Y_{n}\right)$ 


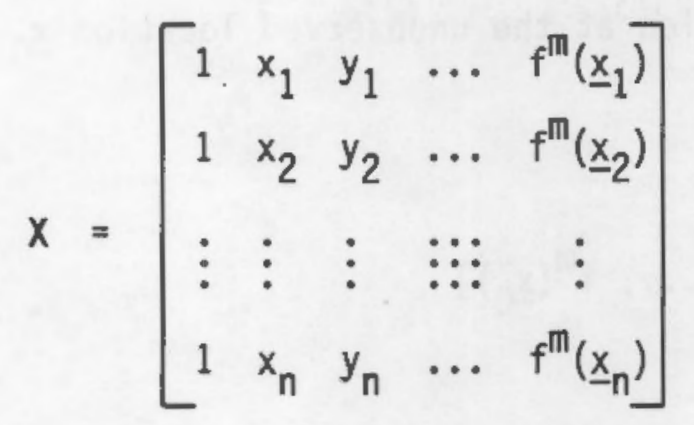

$f^{m}\left(\underline{x}_{i}\right)=$ a low order polynomial of $\underline{x}_{i}$

$$
\begin{aligned}
& \underline{\beta}^{\prime}=\left(\underline{\beta}_{1}, \underline{\beta}_{2}, \ldots, \underline{\beta}_{m}\right) \\
& \epsilon \sim\left(\underline{0}, \sigma^{2} v\right) \text { (i.e., distributed with mean zero and }
\end{aligned}
$$

$$
\begin{aligned}
v & =\left[\begin{array}{cccc}
\sigma\left(\underline{x}_{1} \underline{x}_{1}\right) & \sigma\left(\underline{x}_{1} \underline{x}_{2}\right) & \ldots & \sigma\left(\underline{x}_{1} \underline{x}_{n}\right) \\
\sigma\left(\underline{x}_{2} \underline{x}_{1}\right) & \sigma\left(\underline{x}_{2} \underline{x}_{2}\right) & \ldots & \sigma\left(\underline{x}_{2} \underline{x}_{n}\right) \\
\vdots & \vdots & \vdots: \vdots & \vdots \\
\sigma\left(\underline{x}_{n} \underline{x}_{1}\right) & \sigma\left(\underline{x}_{n} \underline{x}_{2}\right) & \ldots & \sigma\left(\underline{x}_{n} \underline{x}_{n}\right)
\end{array}\right] \\
\sigma^{2}= & \text { the sill of the semi-variogram. }
\end{aligned}
$$

Then

$$
\begin{aligned}
\hat{\beta} & =\left(X^{\prime} V^{-1} X\right)^{-1} X^{\prime} V^{-1} \underline{Y} \\
\hat{\sigma}^{2} & =\frac{1}{n-m}(\underline{Y}-X \hat{\underline{\beta}})^{\prime} V^{-1}(\underline{Y}-X \hat{\underline{\beta}}) \\
\operatorname{VAR}[\hat{\underline{\beta}}] & =\sigma^{2}\left(X^{\prime} V^{-1} X\right)^{-1} .
\end{aligned}
$$


Now, the value of the realization at the unobserved location $\underline{x}_{0}$ is

$$
Y_{0}=\underline{x}_{0}^{\prime} \underline{\beta}+\epsilon_{0}
$$

where $\quad \underline{x}_{0}^{\prime}=\left[1, x_{0}, y_{0}, \ldots, f^{m}\left(\underline{x}_{0}\right)\right]$

$$
\begin{aligned}
\epsilon_{0} & \sim\left(0, \sigma^{2}\right) \\
\operatorname{cov}\left[\epsilon_{1} \epsilon_{0}\right] & =\sigma^{2} \underline{v}_{0} \\
\underline{v}_{0}^{\prime} & =\left[\sigma\left(\underline{x}_{1}, \underline{x}_{0}\right), \sigma\left(\underline{x}_{2}, \underline{x}_{0}\right), \ldots, \sigma\left(\underline{x}_{n}, \underline{x}_{0}\right)\right] .
\end{aligned}
$$

Then,

$$
\left[\begin{array}{c}
\epsilon_{0} \\
\epsilon
\end{array}\right] \sim\left(\left[\begin{array}{l}
0 \\
\underline{0}
\end{array}\right], \sigma^{2}\left[\begin{array}{ll}
1 & \underline{v}_{0}^{\prime} \\
\underline{v}_{0} & v
\end{array}\right]\right)
$$

so

$$
\epsilon_{0} \mid \epsilon \sim\left(\underline{v}_{0} \cdot v^{-1} \epsilon, \sigma^{2}\left(1-\underline{v}_{0}^{\prime} v^{-1} \underline{v}_{0}\right)\right)
$$

Thus,

$$
\hat{Y}_{0}=\underline{x}_{0}^{\prime} \hat{\underline{\beta}}+\hat{\epsilon}_{0}
$$

where

$$
\hat{\epsilon}_{0}=\underline{v}_{0} \cdot v^{-1} \hat{\epsilon} \text {. }
$$

Then,

$$
\begin{aligned}
& \hat{y}_{0}=\underline{x}_{0} \cdot \hat{\underline{\beta}}+\underline{v}_{0} \cdot v^{-1} \hat{\epsilon} \\
& =\underline{x}_{0} \hat{\beta}^{\prime}+\underline{v}_{0}{ }^{\prime} v^{-1}(\underline{Y}-\underline{x} \hat{\underline{\beta}}) \\
& =\underline{v}_{0} \cdot v^{-1} \underline{Y}+\underline{x}_{0} \cdot \hat{\underline{\beta}}-\underline{v}_{0} \cdot v^{-1} x \hat{\underline{\beta}} \\
& =\underline{v}_{0}{ }^{\prime} v^{-1} \underline{Y}+\left(\underline{x}_{0}{ }^{\prime}-\underline{v}_{0} \cdot v-1 x\right) \hat{\beta} \\
& =\underline{v}_{0} \cdot v^{-1} \underline{Y}+\left(\underline{x}_{0}{ }^{\prime}-\underline{v}_{0} \cdot v^{-1} X\right)\left(X^{\prime} v^{-1} x\right)^{-1} X^{\prime} v^{-1} \underline{Y} \\
& =\left[\underline{v}_{0}{ }^{\prime} v^{-1}+\left(\underline{X}_{0}^{\prime}-\underline{v}_{0}^{\prime} v^{-1} X\right)\left(X^{\prime} V^{-1} X\right)^{-1} X^{\prime} V^{-1}\right] \underline{Y} \\
& =\Delta^{\prime} \underline{Y}
\end{aligned}
$$

where $\Delta$ is the vector of kriging weights.

Now, 


$$
\begin{aligned}
& \operatorname{VAR}\left[\hat{Y}_{0}\right]=\operatorname{VAR}\left[\lambda^{\prime} \underline{Y}\right]=\Delta^{\prime} \operatorname{VAR}[\underline{Y}] \Delta=\sigma^{2} \lambda^{\prime} V \Delta \\
& =\sigma^{2} \underline{v}_{0} \cdot v^{-1} \underline{v}_{0}+\sigma^{2}\left(\underline{x}_{0}{ }^{\prime}-\underline{v}_{0} v^{-1} X\right)\left(X^{\prime} v^{-1} X\right)^{-1}\left(\underline{x}_{0}^{\prime}-\underline{v}_{0}^{\prime} v^{-1} X\right)^{\prime} \\
& +2 \sigma^{2} \underline{v}_{0} v^{-1} x\left(x^{\prime} v^{-1} x\right)^{-1}\left(\underline{x}_{0}^{\prime}-\underline{v}_{0}^{\prime} v^{-1} x\right)^{\prime} \\
& \operatorname{VAR}\left[Y_{0}\right]=\sigma^{2}
\end{aligned}
$$

and

$$
\begin{aligned}
\operatorname{cov}\left[\hat{Y}_{0}, \underline{Y}_{0}\right] & =\operatorname{cov}\left[\lambda^{\prime} \underline{Y}_{,} \underline{Y}_{0}\right]=\Delta^{\prime} \operatorname{cov}\left[\underline{Y}_{,}, Y_{0}\right]=\sigma^{2} \underline{\Delta}^{\prime} \underline{v}_{0} \\
& =\sigma^{2}\left[\underline{v}_{0}^{\prime} V^{-1}+\left(\underline{X}_{0}^{\prime}-\underline{v}_{0}^{\prime} V^{-1} X\right)\left(X^{\prime} V^{-1} X\right)^{-1} X^{\prime} V^{-1}\right] \underline{v}_{0} \\
& =\sigma^{2} \underline{v}_{0} V^{-1} \underline{V}_{0}+\sigma^{2}\left(\underline{x}_{0}^{\prime}-\underline{v}_{0}^{\prime} V^{-1} X\right)\left(X^{\prime} V^{-1} X\right)^{-1} X^{\prime} V^{-1} \underline{v}_{0}
\end{aligned}
$$

So,

$$
\operatorname{VaR}\left[\hat{Y}_{0}-Y_{0}\right]=\operatorname{var}\left[\hat{Y}_{0}\right]+\operatorname{Var}\left[Y_{0}\right]-2 \operatorname{Cov}\left[\hat{Y}_{0}, Y_{0}\right]
$$

thus,

$$
\begin{aligned}
& \operatorname{VAR}\left[\hat{Y}_{0}-\underline{Y}_{0}\right]=\sigma^{2}-\sigma^{2} \underline{v}_{0} \cdot V^{-1} \underline{v}_{0} \\
& +\sigma^{2}\left(\underline{x}_{0}^{\prime}-\underline{v}_{0}{ }^{\prime} v^{-1} x\right)\left(X^{\prime} v^{-1} x\right)^{-1}\left(\underline{x}_{0}^{\prime}-\underline{v}_{0}^{\prime} v^{-1} x\right)^{\prime} \text {. }
\end{aligned}
$$

This is the kriging variance.

\subsection{WITHIN-SITE VARIATION}

The nugget effect in kriging is often attributed to the within-site variation. The practical result of using the nugget effect in kriging is to force the surface through the observations. That is, the kriging estimate at a site which has an observation is that observation. Thus the surface has "spikes" wherever there are observations. These "spikes" have no surface area associated with them, they are a jump discontinuity at the site of the observation. Additionally, the sites with duplicate 
observations must be preprocessed (usually the mean observation is calculated), since only one observation per site can be used.

In the GLS estimation procedure, the within-site variation is accounted for by an additional parameter in the model,

$$
\underline{Y}=X \underline{\beta}+\underline{\epsilon}+\underline{\eta}
$$

where $\quad \eta \sim\left(0, \sigma_{w}^{2} I\right)$

$\eta$ and $\epsilon$ are uncorrelated.

These two sources of error can be combined so that

$$
\underline{Y}=X \underline{\beta}+\underline{v}
$$

where $\quad v \sim\left(0, \sigma^{2} V+\sigma_{W}^{2} I\right)$.

Then in the derivations shown in Section $3.5, \sigma^{2} v$ is replaced by $\sigma^{2} v+\sigma^{2} I$. Note that $\underline{v}_{0}$ is not changed.

This formulation assumes that the within-site variation is the same for all sites. However, if the information is available, each site could have it's own within-site variation and $\sigma^{2} I$ is simply replaced with a diagonal matrix, with the diagonal elements being the within-site variation at each site.

By using this within-site variation formulation, the only time the estimate will differ from the kriging estimate using the nugget is at the observation. Now the estimate is no longer the same as the observation. Additionally, sites with duplicate observations do not need to be preprocessed. The algorithm in essence uses their mean.

If there is no within-site variation, then the estimate at a site where there is an observation will be that observation. That is, the model assumes that there is no variation (or error) in the data. In the original application of kriging to ore reverse estimation, this assumption may be valid. However, in applying kriging to wet deposition summaries, the 
assumption that the data at a site has no inherent variability or error does not appear to be valid.

\subsection{ADVANTAGES OF GLS APPROACH}

When the variance-covariance matrix is specified using a function that is dependent on distance, the GLS approach is kriging. However, the GLS approach has the flexibility to both investigate the phenomenon of interest and to add additional information to the model.

Often one semi-variogram does not fit the entire region of interest. Currently, to get around this problem, the region is divided into several smaller regions. The boundaries of the sub-regions are usually artificial and ad-hoc procedures must be used to blend the results for the separate sub-regions together. By doing a "moving" GLS, where only a relatively small number of observations near the point of interest are used in the estimation process, the changes in the "sill" of the semi-variogram can be investigated over the region of interest. Additionally, by using a "moving" approach, the problem with the boundaries of the arbitrarily chosen regions no longer exist.

The GLS approach allows other information to be added to the model. For example, there are some pollutants that are highly correlated with population density (e.g. automobile exhausts). Additionally, information about population density across the United States can be obtained or well estimated with census information. By adding this population information to the model, observations in urban areas will not cause overestimation in nearby rural areas and observations in rural areas will not cause underestimation in nearby urban areas. 



\subsection{VARIOGRAM ESTIMATES}

From previous studies it is known that one semi-variogram does not fit the entire region shown in Figure 2.1. The shape of the semi-variograms for $\mathrm{pH}$ and sulfate precipitation concentration and deposition are primarily due to the "depression," for $\mathrm{pH}$, and "hump", for sulfate, in the surface that is centered around northeastern West Virginia. These surfaces maintain about the same slopes within the smaller region shown in Figure 2.1. Outside this region the slopes change markedly (increases with $\mathrm{pH}$ and decreases with sulfate) and thus the semi-variogram model also changes.

Because our concern in this paper is about the effects of changing criteria for data, we will only look at the region where one variogram reasonably works. Because the exact boundary for this region is not known, the semi-variograms are calculated using only the sites within the smallest region. However, the grid is expanded to the larger region and any site shown in Figure 2.1 is potentially used in the estimate.

Figure 4.1 shows the raw semi-variograms and the estimated models for $\mathrm{pH}$, sulfate concentration and sulfate deposition. As seen in this figure the semi-variograms do not change very much between the four different subsets. Therefore, one semi-variogram model can be used for all four subsets. The "nugget" that is observed at the origin of these semivariograms is used as the estimates of the within-site variation. Additionally, since we use the GLS method the semi-variogram model is converted to it's covariance equivalent (see equation 8 ). The $\mathrm{pH}$ has a linear model with a sill of 0.03 ( $\mathrm{pH}$ units) ${ }^{2}$, a range of 1280 kilometers and a within-site variance of 0.002 ( $\mathrm{pH}$ units) ${ }^{2}$. The sill of the linear model is artificially set, beyond the actual range used, since the covariance model needs a sill. The sulfate concentration has an exponential model with a sill of $0.44,(\mathrm{mg} / 1)^{2}$ a range of $1150 \mathrm{kilometers}$ and a within-site variance of $0.02(\mathrm{mg} / 1)^{2}$. The sulfate deposition has an exponential model with a sill of $0.70\left(\mathrm{~g} / \mathrm{m}^{2}\right)^{2}$, a range of $960 \mathrm{kilometers}$ and a within-site variance of $0.03\left(\mathrm{~g} / \mathrm{m}^{2}\right)^{2}$. The exponential model actually 
never reaches the sill, the range given above is the distance where the model is $95 \%$ of the sill. 

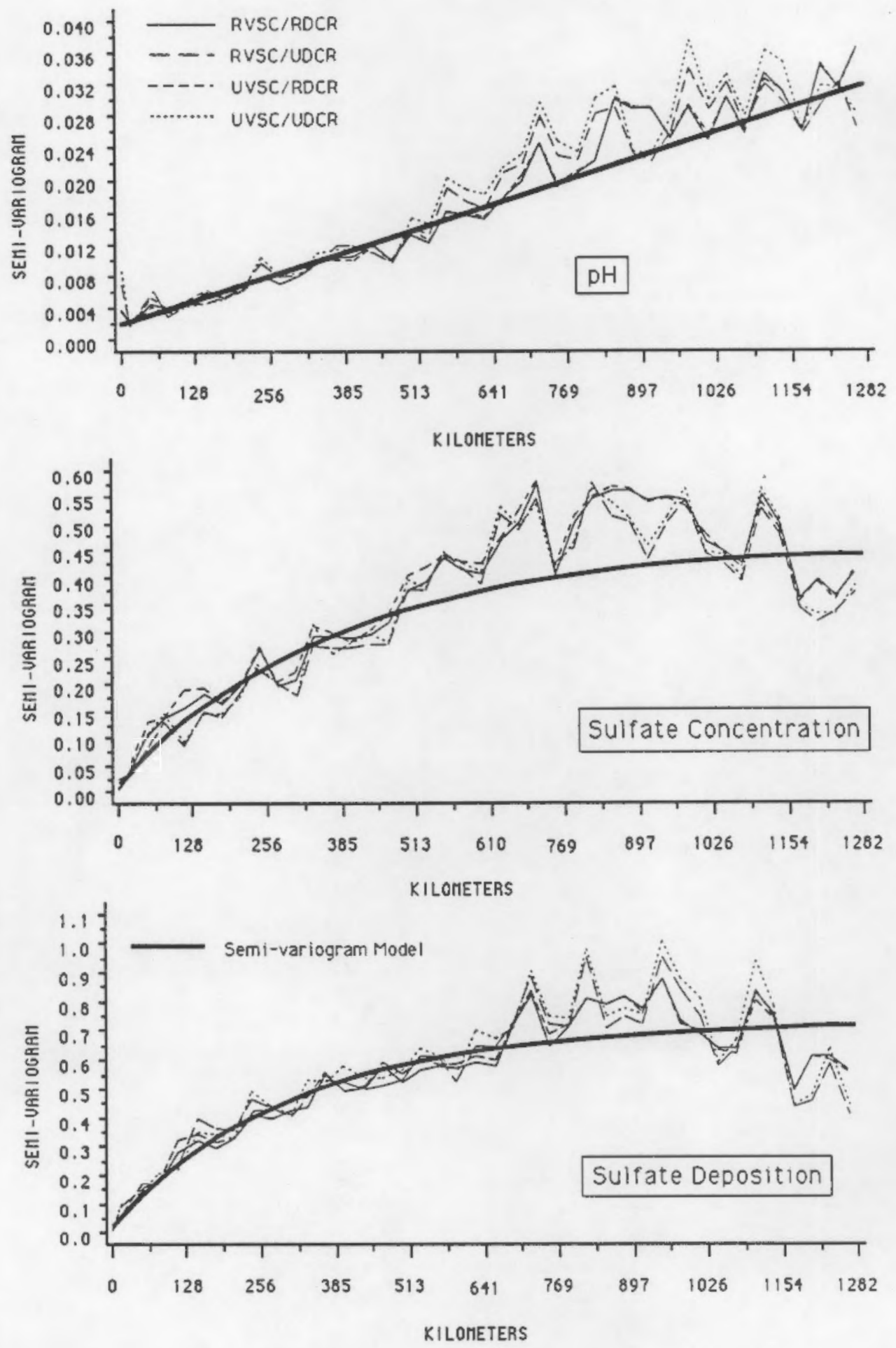

FIGURE 4.1. Semi-variogram for Each of the Subsets of pH, Sulfate Concentration and Sulfate Distributions 



\subsection{RESULTS}

The GLS variation of kriging, described in Section 3, is used to estimate the $\mathrm{pH}$, sulfate concentration, and sulfate deposition for each of the subsets at grid nodes of a regular square grid. This procedure uses the eight closest sites to the grid node in the estimate. The grid nodes are 32 kilometers apart and the area of the grid, shown in Figure 2.1, consists of 3669 nodes. This grid is then contoured using bilinear interpolation in SAS (procedure gcontour).

The contour maps of the $\mathrm{pH}$ and sulfate concentration and deposition show, in broad terms, the effects of using different subsets of the observations. Additionally, contour maps of the differences between several of the different subsets at each grid node are prepared. These maps show the "local" effects, both extent and magnitude, of using different subsets of observations.

\subsection{CONTOUR MAPS OF THE ESTIMATES}

Figures 5.1, 5.2, and 5.3 show the contoured estimates of $\mathrm{pH}$, sulfate concentration and deposition, respectively, using the UVSC/UDCR subsets of observations. These maps are shown for two reasons. First, they are the maps that use the observations that meet the current, and most stringent, sample validity and data completeness criteria. Thus, the effects of using different subsets of observations are judged relative to these maps. Second, these maps are large enough to include details, such as the contour levels, that become lost as the maps are reduced in size for comparisons. As seen in Figures 5.1 and 5.2, the $\mathrm{pH}$ and sulfate concentrations have relatively smooth contour maps. However, as seen in Figure 5.3, sulfate deposition's contour map has several mounds and depressions. The sulfate deposition is the multiple of the sulfate concentration and total precipitation. The mounds and depressions in the sulfate deposition contour map are due to precipitation gradients that are not parallel to the concentration gradients or to local precipitation that is unusually high or low compared to neighboring sites. 


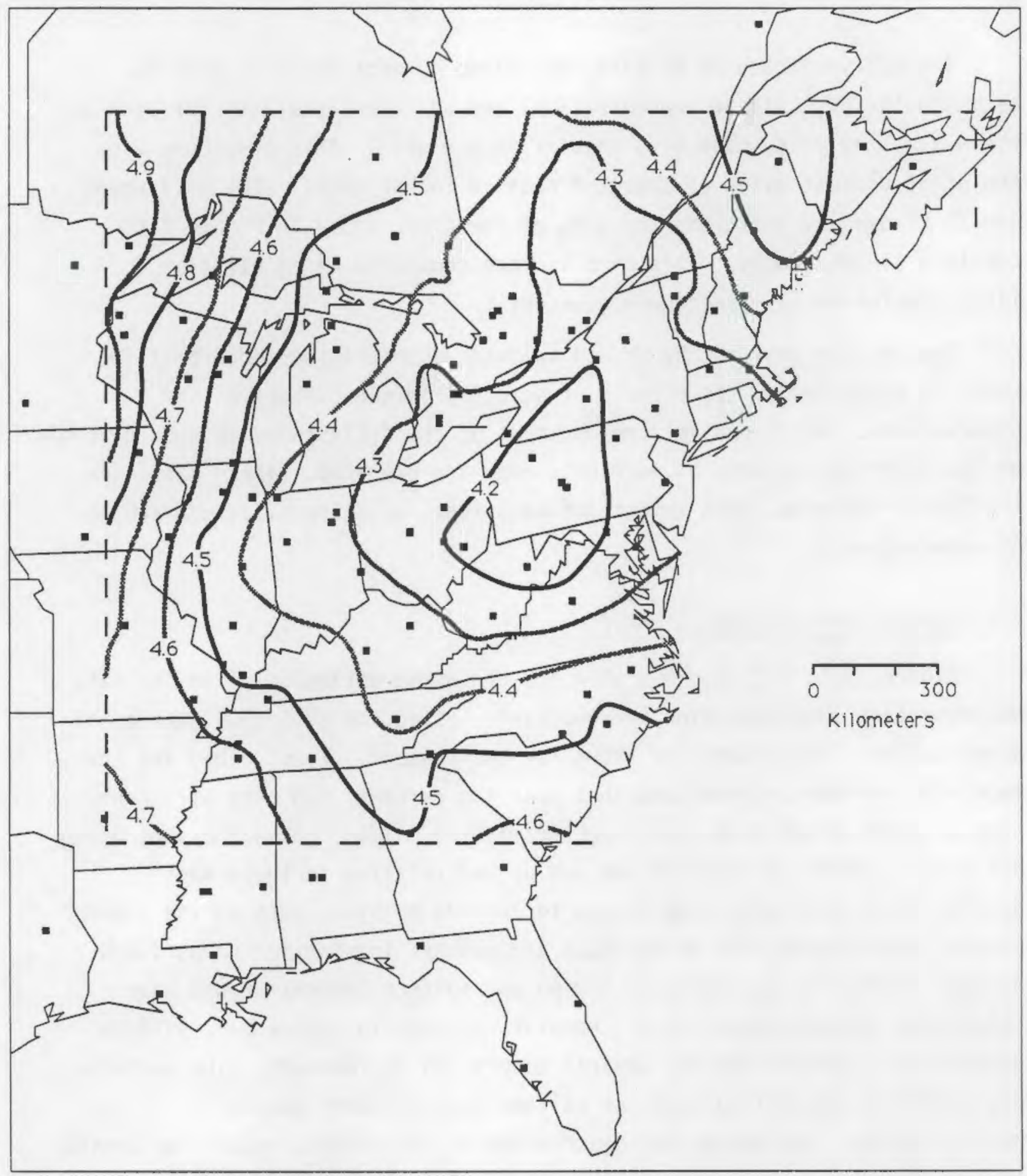

FIGURE 5.1. Contours of pH Estimates Using the UDDC Valid Sample Criteria/UDDC Data Completeness Rating Subset 


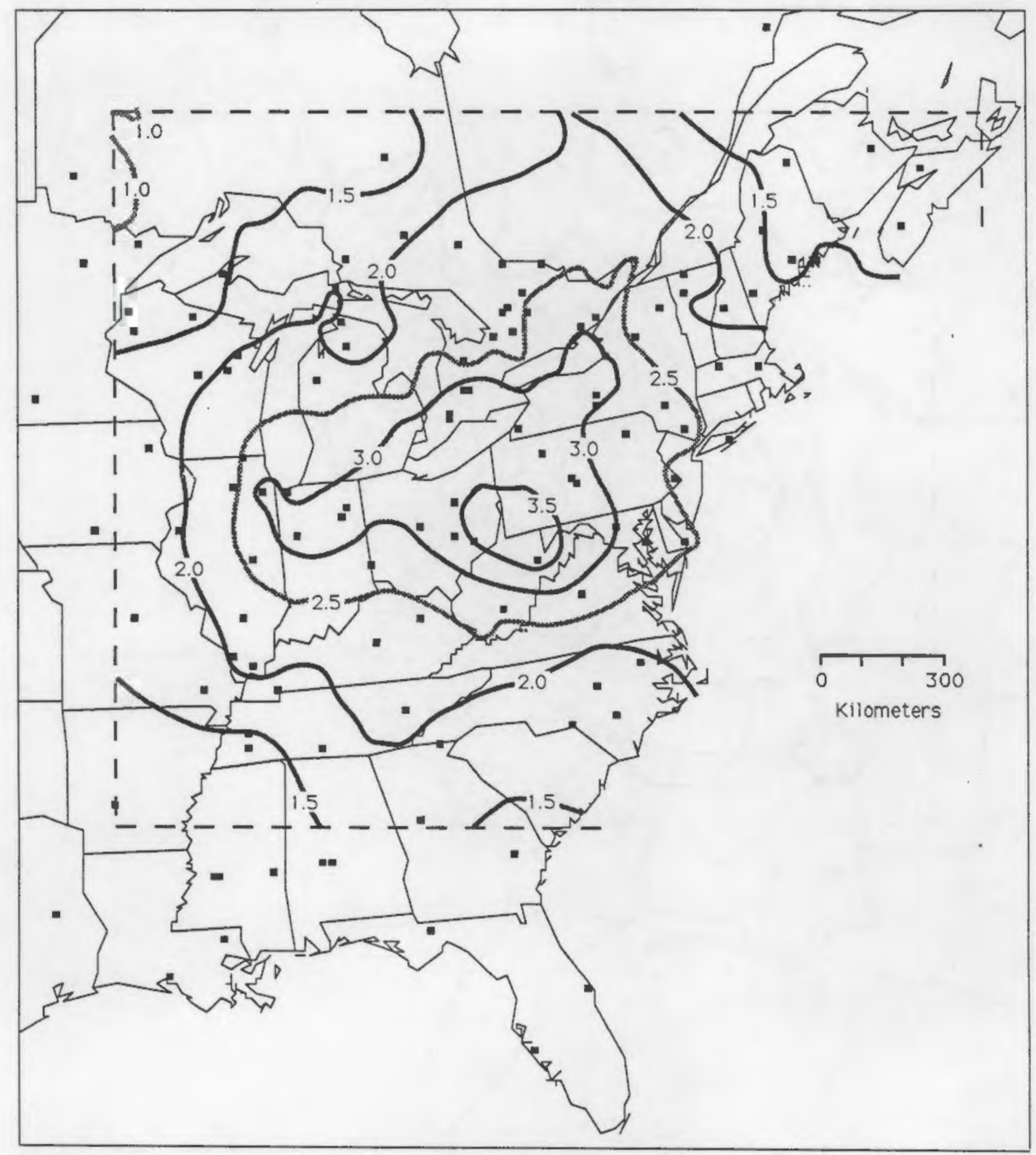

FIGURE 5.2. Contours of Sulfate Concentrations (mg/1) Estimates Using the UDDC Valid Sample Criteria/UDDC Data Completeness Rating Subset 


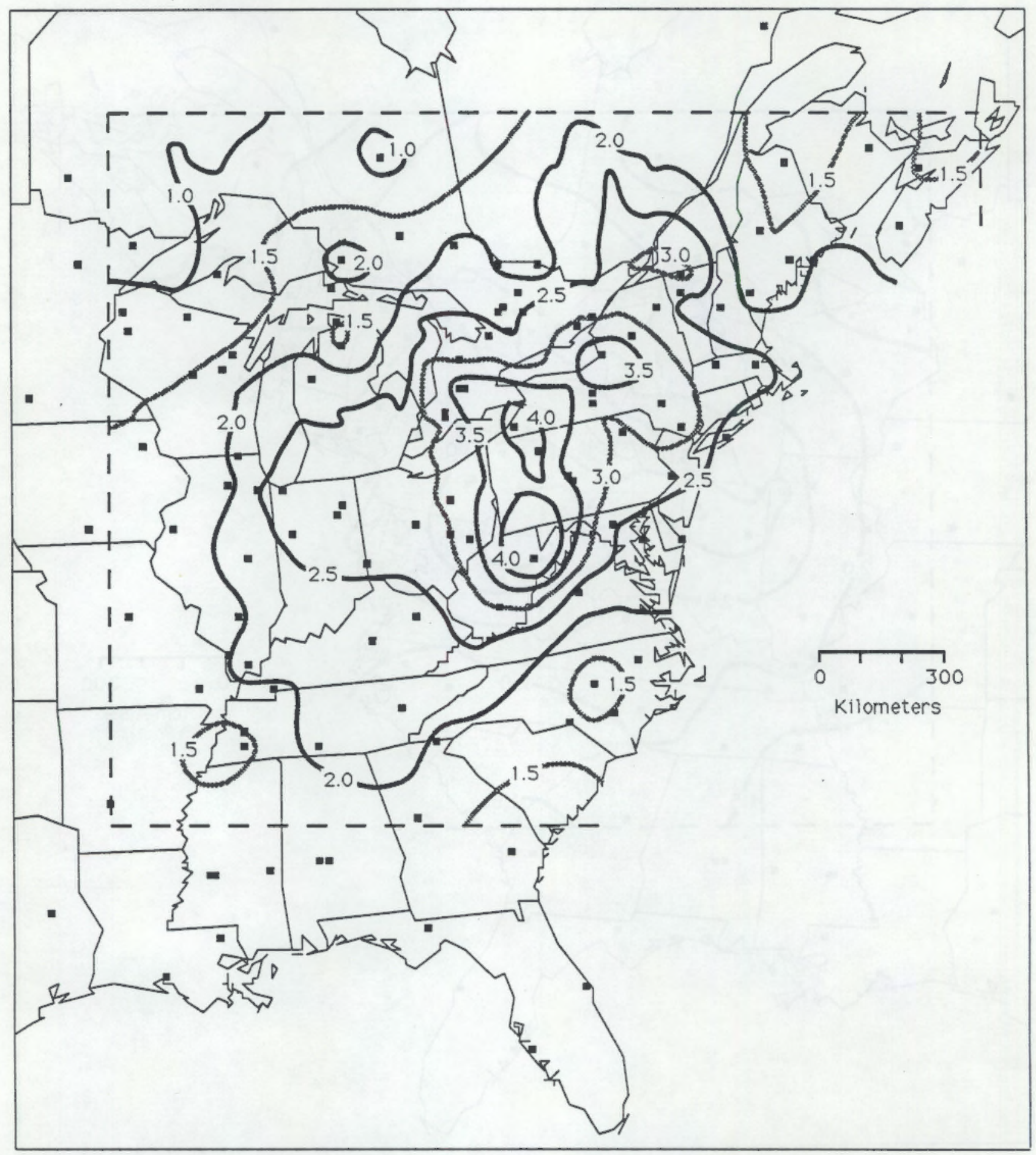

FIGURE 5.3. Contours of Sulfate Depositions $(\mathrm{g} / \mathrm{sq} \mathrm{m})$ Estimates Using the UDDC Valid Sample Criteria/UDDC Data Completeness Rating Subset 
Figures 5.4, 5.5, and 5.6 show the contoured estimates of $\mathrm{pH}$, sulfate concentration and deposition, respectively, using all four subsets of observations. In these figures, the number of sites used increases from left to right (UDCR versus RDCR) and the value of the observations change from top to bottom (UVSC versus RVSC).

The $\mathrm{pH}$ estimates (see Figure 5.4) increase in Ontario and Quebec when the number of sites used increase, from UDCR to RDCR. This region has few sites, so the addition of a few more sites has a profound effect on the contours. The additional sites also produced increases in $\mathrm{pH}$ in northeastern New York, northern Virginia, southern West Virginia, eastern Tennessee, northern Alabama, northern Mississippi and Arkansas decreases in North Carolina, eastern Wisconsin, and southwestern Indiana. Along the border of Pennsylvania and New York the value of the observation at one site (ADS ID 047a; NADP; Jasper, New York) has a profound effect when the UVSC is used versus the RVSC. This site is not in the UVSC/UDCR subset.

The sulfate concentration estimates (see Figure 5.5) change in Ontario and Quebec when the number of sites used increase, from UDCR to RDCR. As with $\mathrm{pH}$ this region has few sites, so the addition of a few more sites has a profound effect on the contours. However, for sulfate concentration there is a decrease to the north and an increase in the south. The increase in sites have a profound effect on the largest contour, $3.5 \mathrm{mg} / 1$. When the UDCR is used, this contour is confined to northern West virginia, southeastern Ohio and southwestern Pennsylvania. However with the additional sites in southern Ontario, when the RDCR is used, this contour now stretches approximately three hundred of kilometers north-northwest into southern Ontario. There are also increases in southern Indiana and western North Carolina and a decrease in northern Virginia. The change in the values of the observations, UVSC versus RVSC, has the effect of increasing the estimates on the southern border between Indiana and Illinois and in Maine. Again site 047a, along the Pennsylvania and New York border also has a noticeable effect in the UVSC/RDCR subset. 

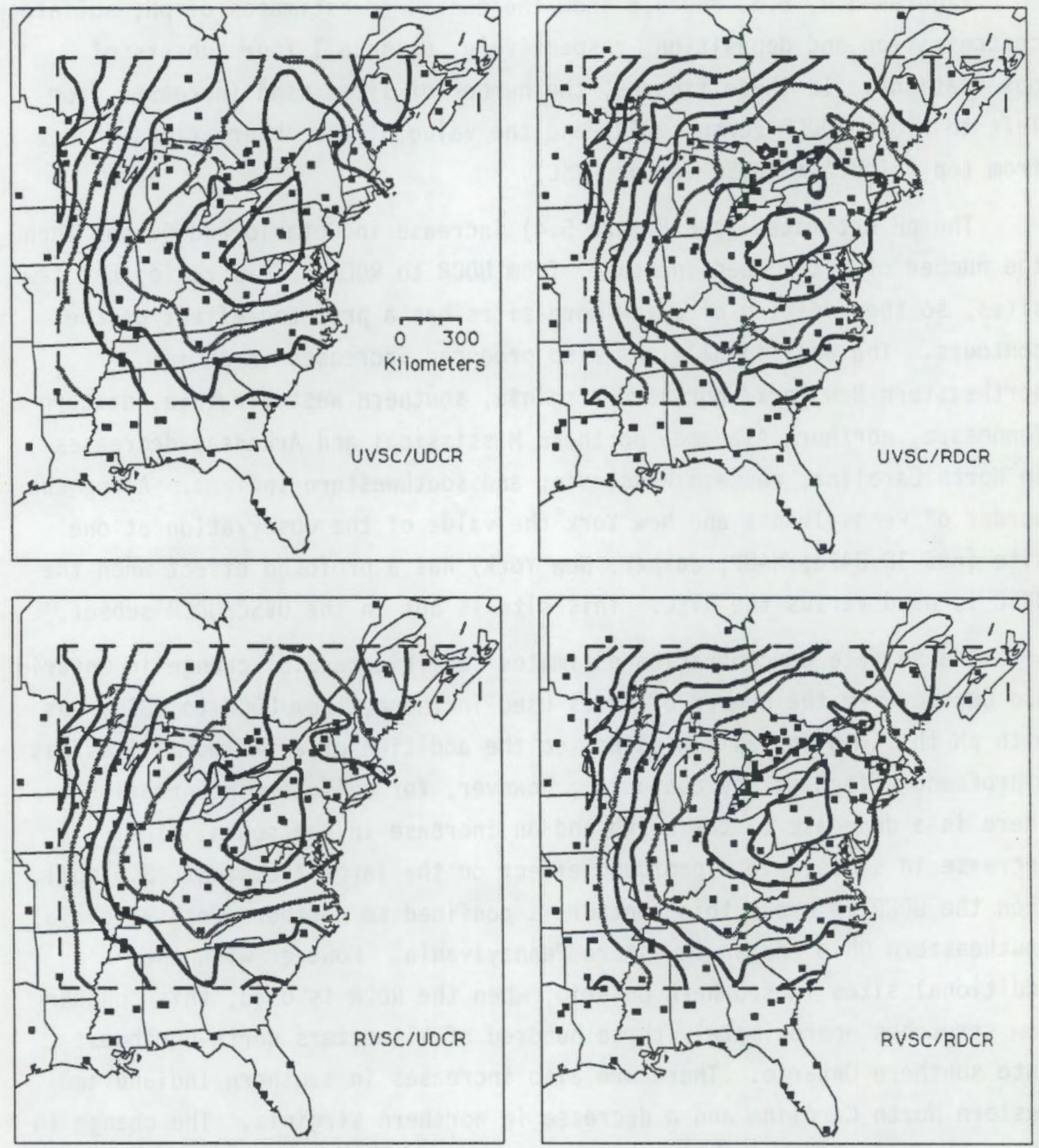

FIGURE 5.4. Contours of $\mathrm{pH}$ Estimates of the Four Subsets Using the UDDC or Relaxed Valid Sample Criteria (UVSC or RVSC) and the UDDC or Relaxed Data Completeness Rating (UDCR or RDCR). The Values of the Contour Lines are Given in Figure 5.1 . 

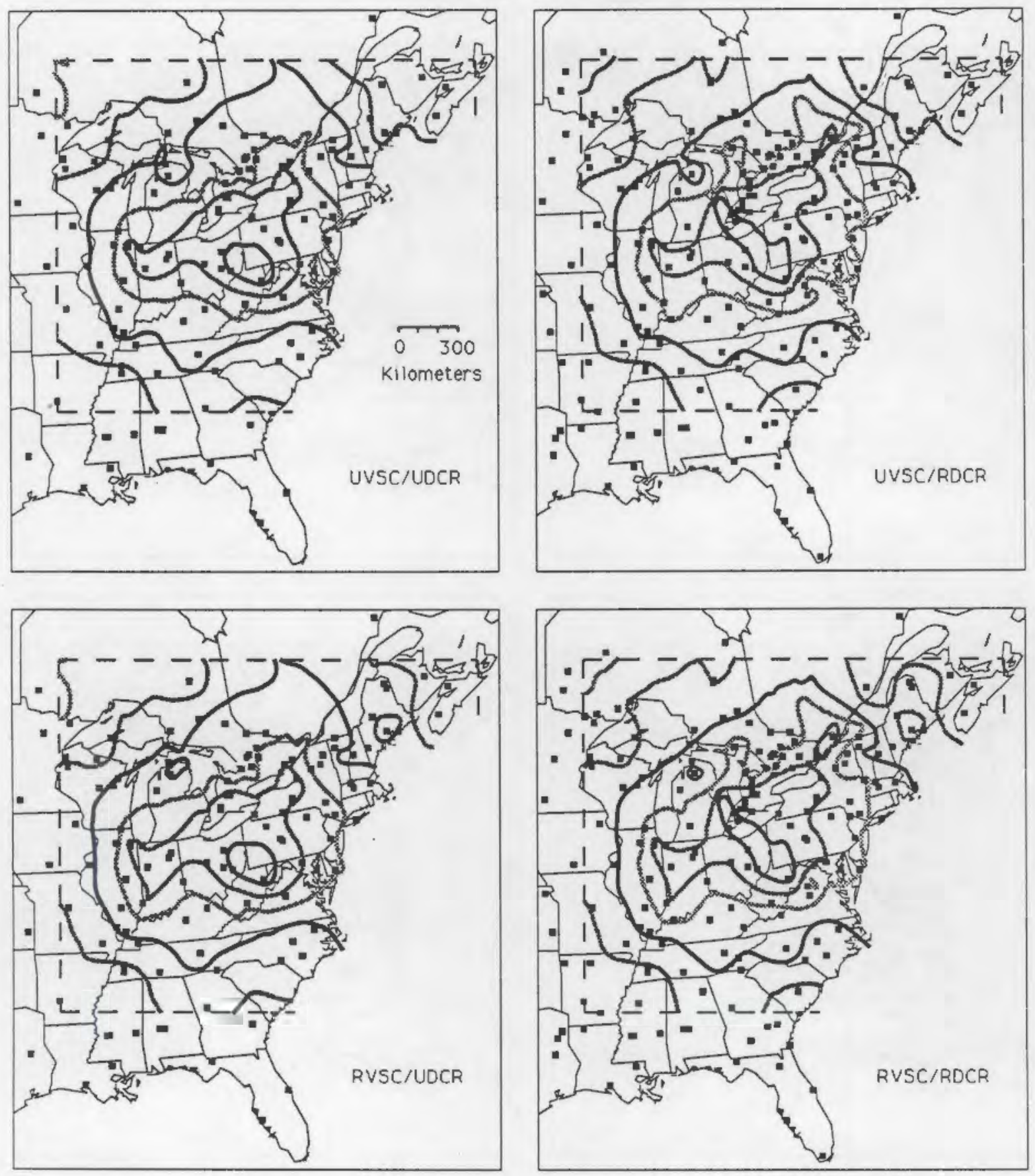

FIGURE 5.5. Contours of Sulfate Concentration (mg/1) Estimates of the Four Subsets Using the UDDC or Relaxed Valid Sample Criteria (UVSC or RVSC) and the UDDC or Relaxed Data Completeness Rating (UDCR or RDCR). The Values of the Contour Lines are Given in Figure 5.2. 

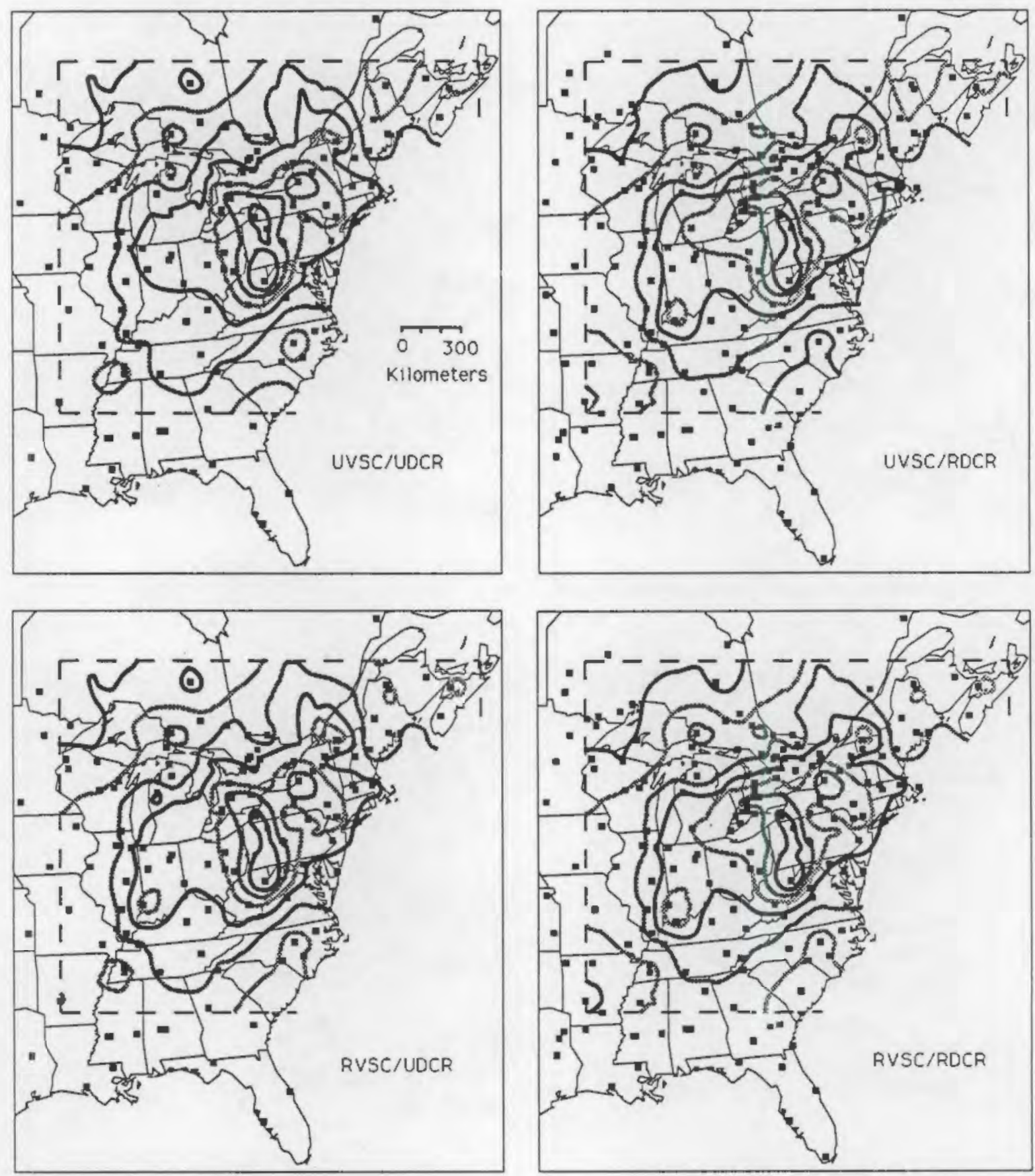

FIGURE 5.6. Contours of Sulfate Deposition $(\mathrm{g} / \mathrm{sq} \mathrm{m})$ Estimates of the Four Subsets Using the UDDC or Relaxed Valid Sample Criteria (UVSC or RVSC) and the UDDC or Relaxed Data Completeness Rating (UDCR or RDCR). The values of the Contour Lines are Given in Figure 5.3. 
The sulfate deposition estimates (see Figure 5.6) increase in southern Ontario, southern Wisconsin and northern Ohio when the number of sites used increase, from UDCR to RDCR. The $3.0 \mathrm{~g} / \mathrm{m}^{2}$ contour moved approximately 300 kilometers west. The presence of two sites on the southwestern border of Indiana bordering on Illinois (ADS ID 420a; NADP; Vincennes, Indiana) and bordering on Kentucky (ADS ID 154a; UAPSP; Rockport, Indiana) caused the $2.5 \mathrm{~g} / \mathrm{m}^{2}$ contour to move approximately 250 kilometers south and an area with a diameter of over 100 kilometers to have depositions greater than $3.0 \mathrm{~g} / \mathrm{m}^{2}$. The increased number of sites also caused decreases in Quebec, Arkansas, northern Mississippi and northern Alabama. The change in the values of the observations, UVSC versus RVSC, has the effect of further increasing the deposition in southwestern Indiana and decreasing the deposition in Maine and New Brunswick.

Figure 5.7 shows the effect of the Parsons, West Virginia site (ADS IO 075a; NADP). The top shows the sulfate concentration and the bottom shows the sulfate deposition, the left side has the contours using the UVSC/UDCR subset while the right side has the contours using the same subset of observations less Parsons, West Virginia. As seen in this figure, the removal of this one unusual site causes reductions in both the concentration and deposition estimates in a five state region (Ohio, Pennsylvania, Maryland, Virginia and West Virginia).

\subsection{CONTOUR MAPS OF THE DIFFERENCES}

Figures 5.8, 5.9, and 5.10 show the contoured differences for selected subsets of $\mathrm{pH}$, sulfate concentration and sulfate deposition, respectively. The upper left map is the difference between the UVSC/UDCR and the RVSC/RDCR subsets. This figure demonstrates the magnitude and extent of the effects of going from the UDDC to the relaxed definitions of both the valid sample criteria and the data completeness ratings. The upper right map is the difference between the UVSC/UDCR and the UVSC/RDCR subsets. The difference in these groups are the additional sites, the values at the sites do not change. The lower left map is the difference between the UVSC/UDCR and the RVSC/UDCR subsets. The primary difference in these groups is the change 

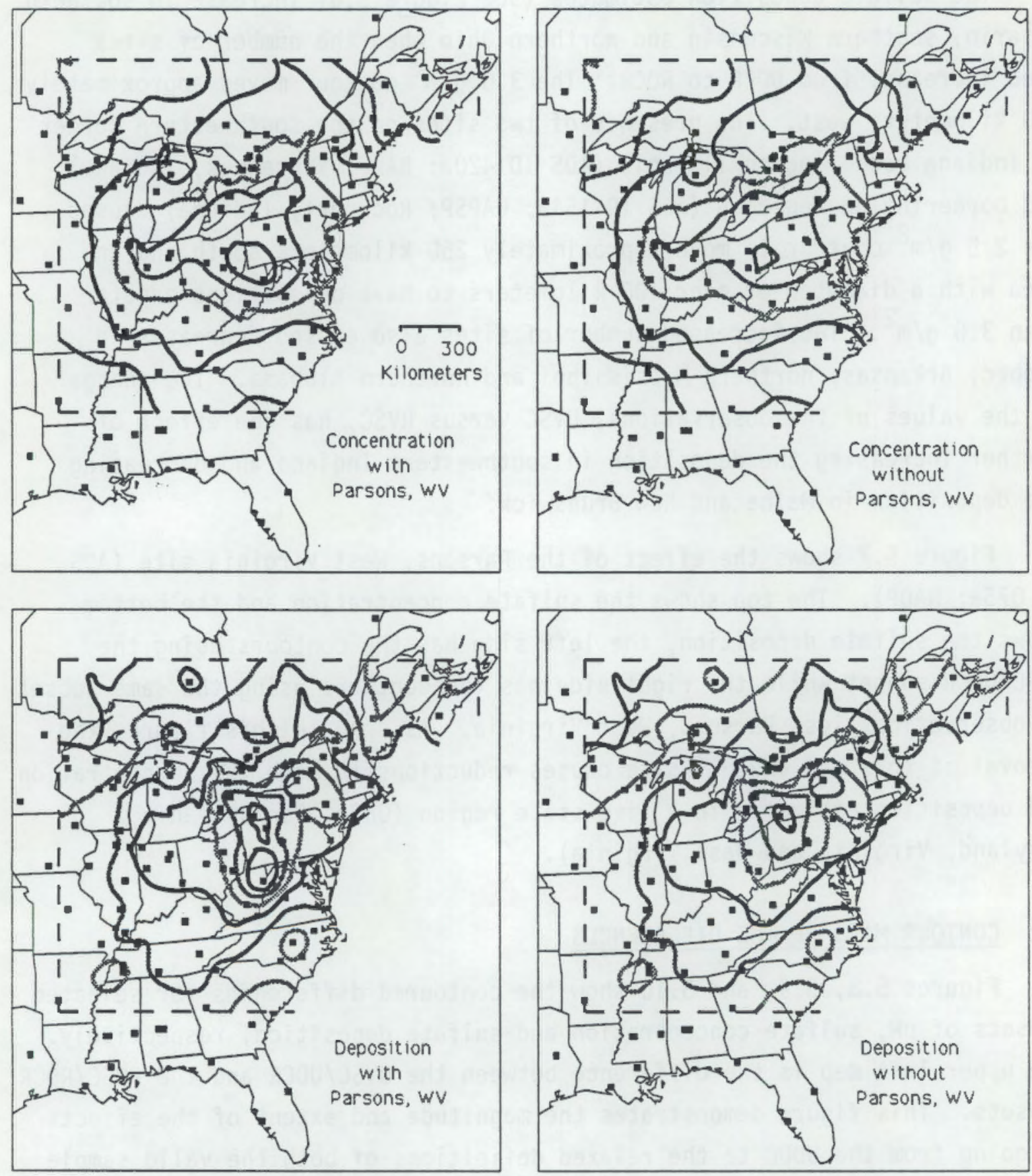

FIGURE 5.7. Comparison of Sulfate Concentration and Deposition Estimates With and Without Parsons, West Virginia Site Present. The Rest of the Sites Belong to UVSC/UDCR Subset. See Figures 5.2 and 5.3 for the Values of the Contour Lines. 

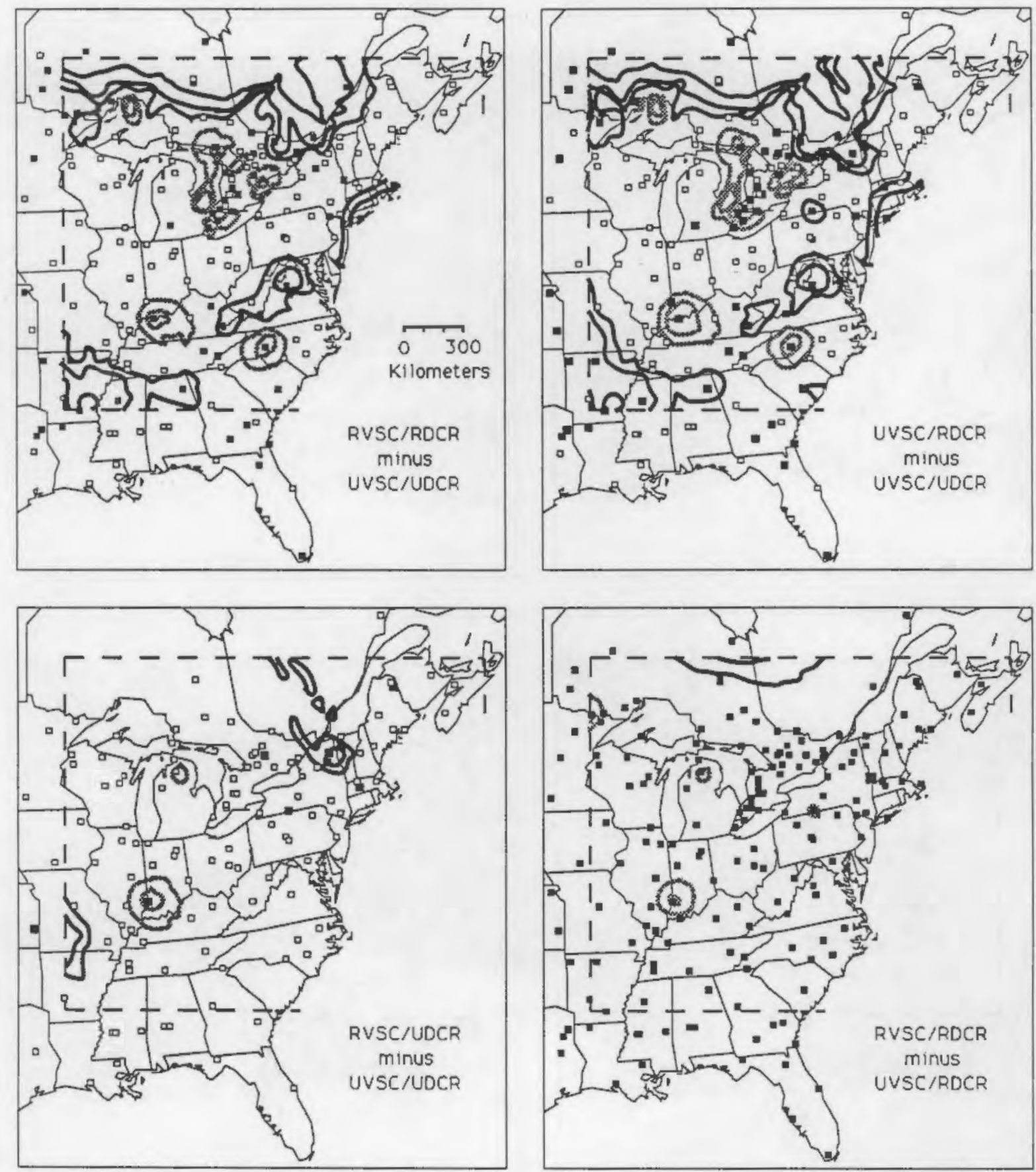

FIGURE 5.8. Differences in pH Estimates for Selected Pairs of Subsets. The Dark Lines Indicate an Increase in the Estimate and the Light Lines Indicate a Decrease in the Estimate. The Outer Contour is 0.025 , the Middle Contour is 0.05 and the Interior Contour is 0.10 . 

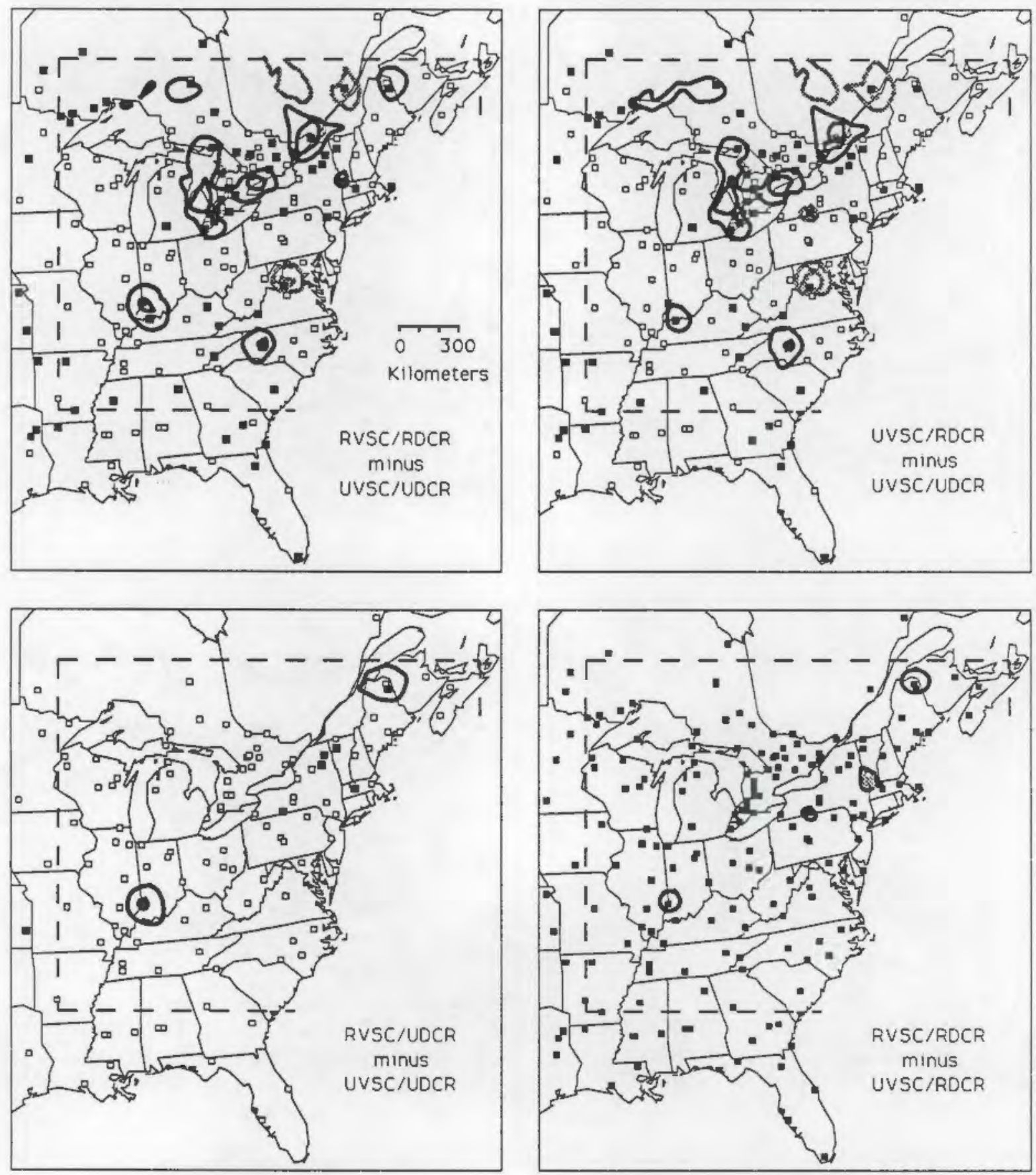

FIGURE 5.9. Differences in Sulfate Concentration Estimates for Selected Pairs of Subsets. The Dark Lines Indicate an Increase in the Estimate and the Light Lines Indicate a Decrease in the Estimate. The Outer Contour is $0.25 \mathrm{mg} / 1$ and the Interior Contour is $0.5 \mathrm{mg} / 1$. 

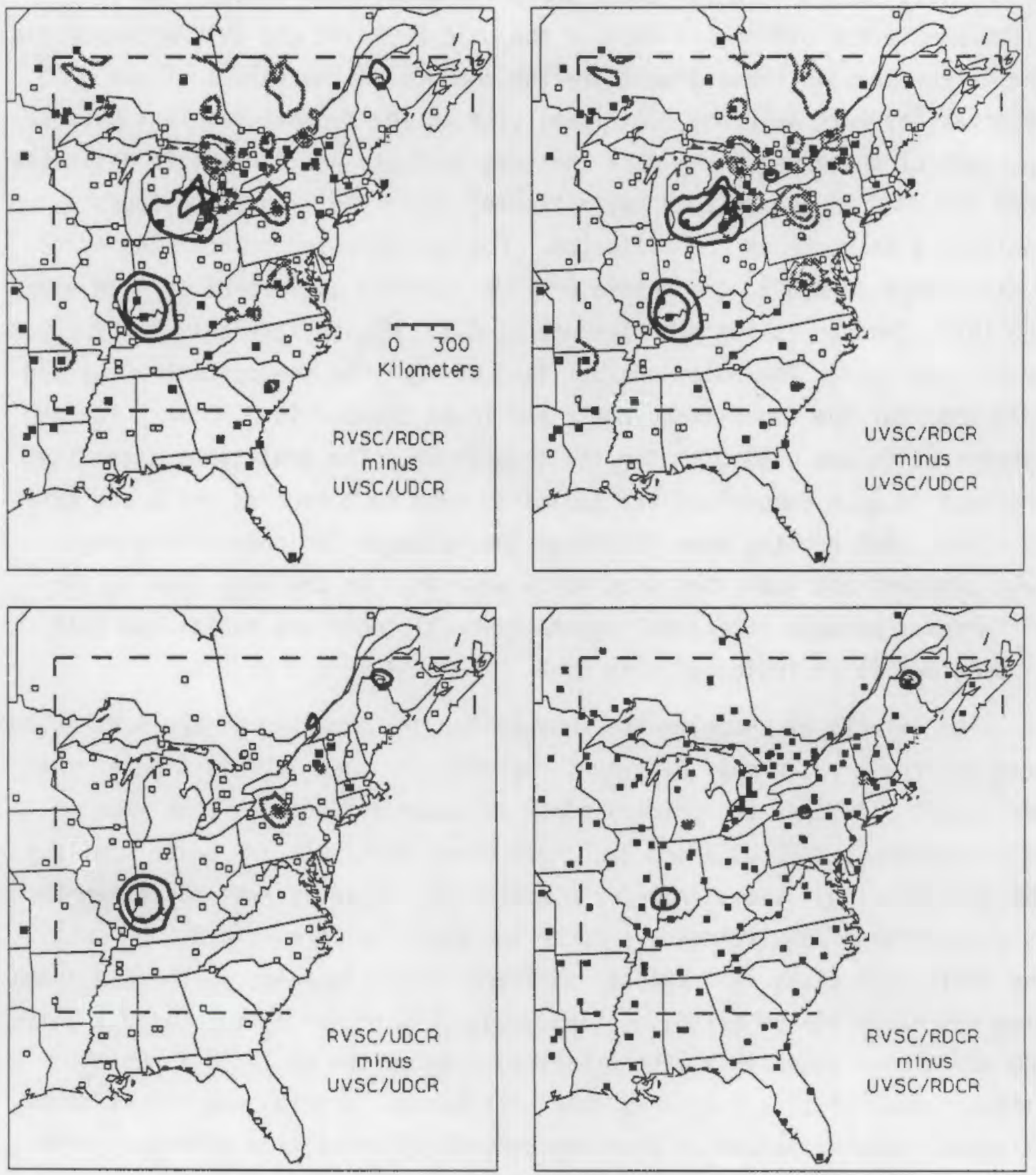

FIGURE 5.10. Differences in Sulfate Deposition Estimates for Selected Pairs of Subsets. The Dark Lines Indicate an Increase in the Estimate and the Light Lines Indicate a Decrease in the Estimate. The Outer Contour is $0.25 \mathrm{~g} / \mathrm{sq} \mathrm{m}$ and the Interior Contour is $0.5 \mathrm{~g} / \mathrm{sq} \mathrm{m}$. 
in the site values, however there are a few additional sites. The lower right map is the difference between the UVSC/RDCR and the RVSC/RDCR subsets. The differences in these groups are the changes in the values of the observations with only one additional site on the Vermont-New York border. For each of these maps, the dark contours indicate an increase in estimates from the UDDC to the relaxed definition(s) while the lighter contours indicate a decrease in the estimates. For $\mathrm{pH}$ the outer contour indicates a difference of 0.025 , the second contour is for a difference of 0.05 and the third contour is for a difference of 0.1 . For sulfate only two contour levels are used. The outer contour is $0.25 \mathrm{mg} / 1$ for the concentration and $0.25 \mathrm{~g} / \mathrm{m}^{2}$ for the deposition, while the inner contour is $0.50 \mathrm{mg} / 1$ for the concentration and $0.50 \mathrm{~g} / \mathrm{m}^{2}$ for the deposition. The additional sites that are used in each comparison are indicated with dark squares while the site locations that are the same (although the value of the observation may have changed) are indicated with white squares. In the maps showing the differences between strict-all and relaxed-all, only one additional site is used and it is indicated with an $x$.

The effects of changing the subsets for $\mathrm{pH}$, shown in Figure 5.8 , extend over a large area in many different regions. As seen in this figure, the additional sites are the primary cause of these differences (the maps of RVSC/RDCR minus UVSC/UDCR and UVSC/RDCR minus UVSC/UDCR are quite similar). The few additional sites in Ontario and Quebec cause an area whose length is greater than 1000 kilometers to be increased by more than $0.1 \mathrm{pH}$ units. The additional sites in Arkansas, northern Mississippi and northern Alabama also increases the $\mathrm{pH}$ estimates throughout this region as much as 0.1 units. The additional sites in southern Ontario reduced the $\mathrm{pH}$ in this region, although much of this region is over Lake Huron. Several additional sites effected "local" regions of over one hundred kilometers in diameter, with several of the regions have considerable area with estimates that changed by more that 0.05 units. Only the five sites with the largest changes in their pH observations (see Table 2.6, sites 047a (Jasper, New York), 208a (Lac Le Croix, Ontario), 241a (Gaylord, Michigan), 420a (Vincennes, Indiana), and 495a (Mooseonee, Ontario)) have a noticeable effect (see 
RVSC/RDCR minus UVSC/RDCR). Of these sites, 495a (Mooseonee, Ontario) which is the northern most site shown in Ontario has the largest effect. The size of this effect is due to the sparsity of data in the region. As seen in Table 2.7, the changes in the values for sites 420a (Vincennes, Indiana) and 495a (Mooseonee, Ontario) are primarily due to one sample with extremely unusual pHs. These samples have extremely poor collection efficiencies $(2.9 \%$ and $2.0 \%$, respectively, of the sample volume predicted from the rain gage is actually present in the collector). Site 047a (Jasper, New York) is very unusual, on one map it causes an increase, on another map it causes a decrease and on the other two maps it has no effect. This site has only one sample that is added (see Table 2.7), however, it is also the only sample in June that is analyzed, thus when the sample is removed, all of June is essentially removed. Without this sample, the $\mathrm{pH}$ value is large compared to it's neighboring sites. When the sample is added, the $\mathrm{pH}$ decreased and the site is no longer large compared to it's neighboring sites.

The effects of changing the subsets for sulfate concentration, shown in Figure 5.9, are greatest in southern Ontario. As seen in this figure, the additional sites are the primary cause of these differences. Most of the new sites are in southern Ontario and have higher concentrations than their neighboring sites. These sites have poor UDDC data completeness ratings and are only included when the data completeness rating in relaxed. Their poor ratings are primarily because of their low collection efficiencies. The three sites with the largest changes in the sulfate concentrations (see Table 2.4, sites 047a (Jasper, New York), 163a (Caribou, Maine) and 420a (Vincennes, Indiana)) have a noticeable effect. The extent of the effects are related to the density of neighboring sites, with the size increasing as the density decreases. The extent of the effect due to site 163a (Caribou, Maine), is effected by site 436a (Presque Isle, Maine) that is just southwest of it. When the UDDC valid sample criteria is used to select the samples, these two sites are similar $(1.32 \mathrm{mg} / 1$ at $163 \mathrm{a}$ verses $1.55 \mathrm{mg} / 1$ at 436a), thus there is no effect on the UVSC/ROCR minus UVSC/UDCR map. When the relaxed valid sample criteria is used to select the samples, 
both site's observations increase $(1.84 \mathrm{mg} / 1$ at $163 \mathrm{a}$ verses $1.70 \mathrm{mg} / 1$ at 436a), thus the extent of the effect shown on the has a RVSC/RDCR minus UVSC/RDCR map has a diameter of a little over 100 kilometers (the averages of these two sites change from $1.44 \mathrm{mg} / 1$ to $1.77 \mathrm{mg} / 1$ ). However on the RVSC/UDCR minus UVSC/UDCR map the diameter of the area effected has increased to over $200 \mathrm{kilometers}$ because the values change from $1.32 \mathrm{mg} / 1$ (163a only) to $1.77 \mathrm{mg} / 1$. The increase in the sulfate concentration at site $163 a$ is due to two samples with unusually high sulfate concentration (52 $\mathrm{mg} / 1$ precipitation weighted average) with very low collection efficiencies ( $11.2 \%$ and $24.8 \%$ ). Sites $420 \mathrm{a}$ along the Indiana-I1linois border (Vincennes, Indiana) and 154a along the Indiana-Kentucky border (Rockport, Indiana) also effect each other. Under the strict criteria for selecting samples their sulfate concentrations are $2.62 \mathrm{mg} / 1$ and $2.65 \mathrm{mg} / 1$, respectively. However only site 154a has an effect when UVSC/RDCR and UVSC/UDCR are compared because of the lower observations to the south of 154a. However when the valid sample criteria is relaxed, site 420a increases to $3.08 \mathrm{mg} / 1$ and causes an effect with a diameter of close to $200 \mathrm{kilometers}$ without the present of $154 \mathrm{a}$ and about $100 \mathrm{kilometers}$ when $154 \mathrm{a}$ is present. The large increase at site $420 \mathrm{a}$ is primarily due to one sample with a concentration of $69 \mathrm{mg} / 1$ and a collection efficiency of only $2.9 \%$. Finally, as with $\mathrm{pH}$, the effects of site 047a (Jasper, New York) change with the different maps and the reasons are the same as before.

The effects of changing the subsets for sulfate deposition, shown in Figure 5.10, are also greatest in southern Ontario. As seen in this figure, the additional sites are again the primary cause of these differences. Unlike the sulfate concentration, not all the effects, in southern Ontario, are an increase in the estimates. While the sulfate concentration (see Figure 5.9) at some sites are high compared to the neighboring sites, their sulfate deposition is low when compared to the same neighboring sites. This anomaly is a result of the very low precipitation at these sites. The effects of sites $154 a$ and $420 a$ in southwestern Indiana are larger than those shown for the sulfate concentration primarily because of the high precipitation at site $154 \mathrm{a}$. These sites together account for an increase 
of $0.5 \mathrm{~g} / \mathrm{m}^{2}$ in sulfate deposition over an area with a diameter of over 200 kilometers. As with $\mathrm{pH}$ and sulfate concentration, site 047 a remains a very unusual site. Although the sulfate concentration increases to a level that is similar to it's neighboring sites, when the valid sample criteria is relaxed, this site has a low precipitation amount as compared to its neighbors. Thus, although the sulfate deposition increase when the valid sample criteria is relaxed, this new value is still low compared to it's neighboring sites.

The extent and magnitude of effect that the Parsons, West Virginia site has on the "local" sulfate concentration and deposition estimates are shown in Figure 5.11. The extent of this effect is over a region with a diameter of approximately 400 kilometers. This region is limited by the use of only the eight closest sites in the estimation process. The magnitude of the effect increases to over $0.5 \mathrm{mg} / 1$ for the concentration and $1.5 \mathrm{~g} / \mathrm{m}^{2}$ for the deposition. The region of those magnitude's of effect are approximately 100 kilometers. The magnitude of the effect of the sulfate deposition is much greater than that for the sulfate concentration because of the high precipitation at this site. 

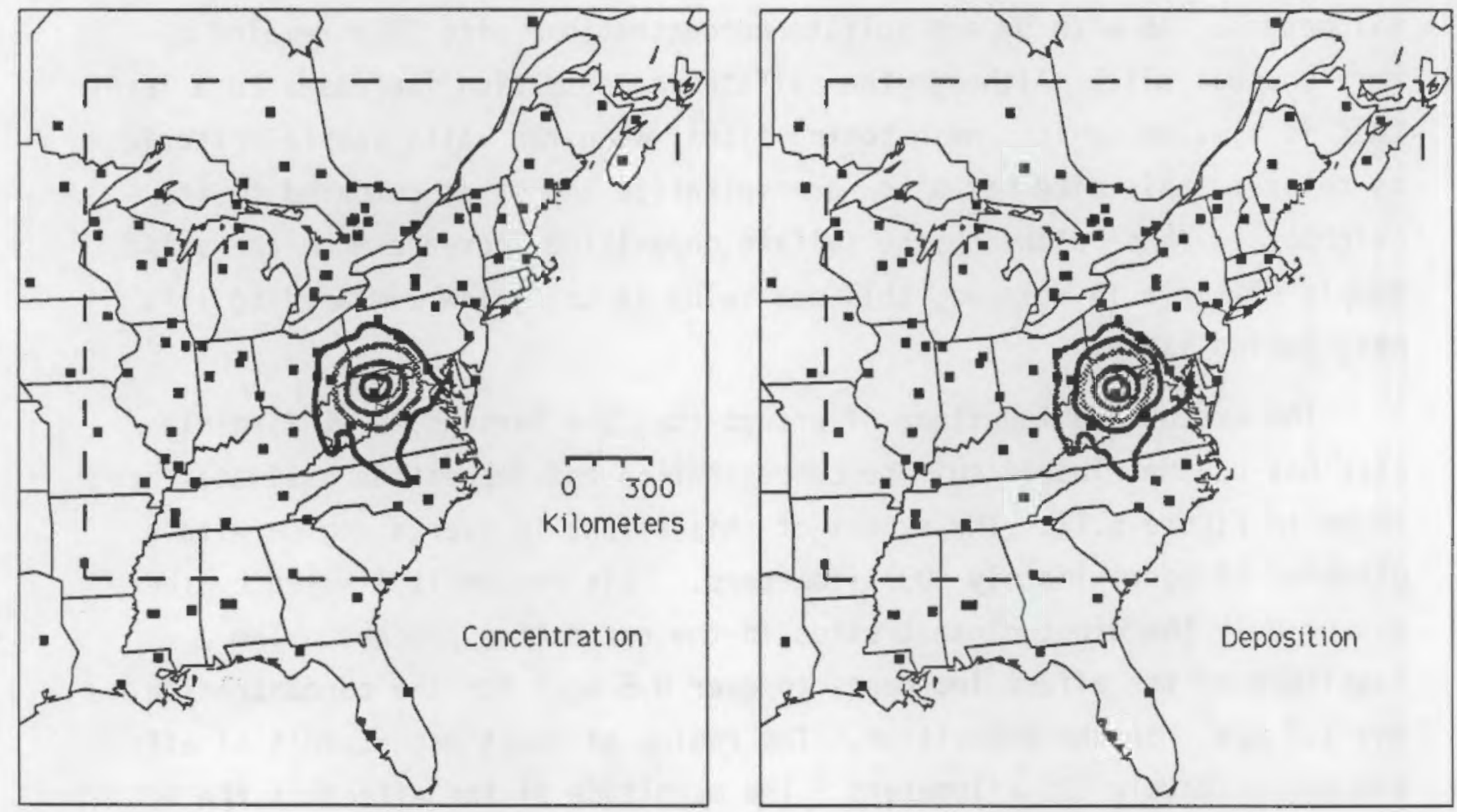

FIGURE 5.11. Extent and Magnitude of Effect the Parsons, West Virginia Site has on the Sulfate Concentration and Deposition Estimates. The Concentration Contours are $0.5,0.25,0.10$ and $0.001 \mathrm{mg} / 1$ and the Deposition Contours are 1.5, 1.0, $0.5,0.25$, and $0.001 \mathrm{~g} / \mathrm{sq} \mathrm{m}$. 


\subsection{CONCLUSIONS}

Application of UDDC valid sample criteria requires that the UDDC rating be used to ensure representativeness throughout all seasons. This is a conservative position for inclusion of data for maps. As seen with site $047 a$ and other sites shown in Table 2.7, when the UDDC valid sample criteria is used, blocks of data over a period of greater than a month may be lost. When a site has seasonal trends, the loss of that much data can adversely effect the annual estimates.

Relaxed valid sample criteria may or may not lead to representative annual summaries for the year. Sites must be evaluated with respect to other nearby sites or years. Seasonal criteria may not guarantee representativeness. That is, using the UDOC data completeness rating requirement does not protect you. As seen in Table 2.7, at a number of sites, the relaxed valid sample criteria let a number of extremely unusual samples to be included in the annual estimates. However, all these samples have very small collection efficiencies. Possibly, the collection efficiency needs to be examined on a sample by sample basis, instead of seasonally and annually.

Representativeness of a site for its surrounding area is very important with a sparse network. Does the sulfate concentration and deposition at the Parsons, West Virginia site represent the area within 200 kilometers of it?

Although the relaxed data completeness rating allows the number of sites used to increase by over $50 \%$, except for a few sites, these additional sites did not change the spatial patterns of wet deposition on a region scale. Most of the changes due to the additional sites are on a local scale where these differences are smaller than the scale used in regional isopleth maps. The few additional sites which do have a profound effect are located in areas where there is a sparsity of sites (e.g. northern Ontario) or whose surmaries are changed markedly due to either the addition of an extremely unusual sample or the addition of a large contiguous number of samples. Thus, for contour maps whose objective is to show regional 
pattern, the key issue is not the number of sites but the location of the site and the validity of the samples from the site.

There are two sources of uncertainty in the contour maps that need to be addressed. First, the within-site variation in the annual summaries. Because there are so few collocated sites, the within-site variation is poorly estimated and thus any variance estimate from kriging is also poorly estimated. Second, the variation from year to year in the annual summaries at a site have not been considered. For example, at Parsons, West Virginia the annual sulfate depositions $\left(\mathrm{g} / \mathrm{m}^{2}\right)$ are $4.4(1979), 4.4(1980), 4.6$ (1981), 4.0 (1982), 3.1 (1983), 3.4 (1984), 4.8 (1985), 5.3 (1986) and 3.0 (1987). It should be noted that in 1985 and $1986,19 \%$ and $9 \%$, respectively, of the precipitation has no chemistry results for sulfate. This occurs primarily in months when the sulfate concentration is low at this site (late fall). Additionally, in 1987 the annual precipitation was only $73 \%$ of the average annual precipitation for the previous eight years.

For network operations, the implication of this study is that sites must give valid data for the entire year. The loss of a large number of contiguous samples renders the site useless for the purpose of annual summaries. The relationship between small sample collection efficiency and the representativeness of the sample's chemistry needs to be reevaluated.

For network design, the implication of this study is that the total number of sites is less important than the representativeness of the site. When considering a region the size of the eastern United States, where the sheer magnitude of the region forces sites to be hundreds of kilometers apart, the summaries from one site can profoundly impact a large region. If that site is not representative of the region between sites, then it will bias the results. 


\subsection{REFERENCES}

Barchet, W. R. 1987. "Acid Deposition and its Gaseous Precursors." Chapter 5 in NAPAP Interim Assessment, the Causes and Effects of Acidic Deposition, volume III: Atmospheric Processes and Deposition. U.S. Government Printing Office, Washington, DC.

Barrie, L. A. and J. M. Hales. 1984. "The Spatial Distributions of Precipitation Acidity and Major Ion Wet Deposition in North America During 1980." Tellus 36B:333-355.

Bilonick, R. A. 1985. "The Time-Space Distribution of Sulfate Deposition in the Northeastern United States." Atmos. Environ. 19(11):1829-1845.

Calvert, J., J. N. Galloway, J. M. Hales, G. M. Hidy, J. Jacobson, A. Lazrus, J. Miller, V. Mohnen, and M. F. Uman. 1983. Acid Deposition, Atmospheric Processes in Eastern North America. National Academy Press, Washington, D.C.

Cowling, E. B. 1982. "Acid Precipitation in Historical Perspective." Environ. Sci. Tech. 16(2):110A-123A.

Eynon, B. P., and P. Switzer. 1983. "The Variability of Rainfall Acidity." Canadian J. Statistics 11(1):11-24.

Finkelstein, P. 1984. "The Spatial Analysis of Acid Precipitation Data." J. Climate and Applied Met. 23:52-62.

Gibson, J. H., J. C. Moore, T. C. Haas, and P. Chapman. 1989. The Use of Kriging to Estimate Wet Deposition Over the Conterminous U.S. Prepared for the U.S. Environmental Protection Agency, Research Triangle Park, North Carolina under Grant No. CR813673-01-1.

Guertin, K., J. -P. Villeneuve, S. Deschenes, and G. Jaques. 1988. "The Choice of Working Variables in Geostatistical Estimation of Acid Precipitation." Atmos. Environ. 22(12):2787-2801.

Junge, C. E. 1963. Air Chemistry and Radioactivity, International Geophysics Series, Vol. 4, pp. 311-346. Academic Pres, New York.

Le, D. N., and J. Petkau. 1988. "The Variability of Rainfall Acidity Revisited." Canadian J. Statistics 16(1):15-38.

Munger, J. W., and S. J. Eisenreich. 1983. "Continential-Scale Variations in Precipitation Chemistry." Environ. Sci. Tech. 17(1):32A-42A.

NADP. 1987. NADP/NTN Annual Data Summary, Precipitation Chemistry in the United States. National Atmospheric Deposition Program Coordinator's 
Office, Natural Resource Ecology Laboratory, Colorado State University, Fort Collins, Colorado.

01sen, A. R. 1989. 1986 Wet Deposition Temporal and Spatial Patterns in North America. PNL-6 933 , Pacific Northwest Laboratory, Richland, Washington.

Olsen, A. R., D. S. Bieglow, W. H. Chan, T. L. Clark, M. A. Lusis, P. K. Misra, R. J. Vet, and E. C. Voldner. 1990. "Unified Wet Deposition Data Summaries for North America: Data Summary Procedures and Results for 1984." Atmospheric Environment, in press.

01sen, A. R., and A. L. Slavich. 1986. Acid Precipitation in North America: 1984 Annual Data Summary from ADS Data Base. EPA-600/4-86-033, U.S. Environmental Protection Agency, Research Triangle Park, North Carolina.

01sen, A. R., and A. L. Slavich. 1985. Acid Precipitation in North America: 1983 Annual Data Summary from ADS Data Base. EPA-600/4-85-061, U.S. Environmental Protection Agency, Research Triangle Park, North Carolina.

01sen, A. R., and C. R. Watson. 1984. Acid Precipitation in North America: 1980, 1981, and 1982 Annual Data Summaries Based on ADS Data Base. EPA-600/7-84-097, U.S. Environmental Protection Agency, Research Triangle Park, North Carolina.

Seilkop, S. K., and P. L. Finkelstein. 1987. "Acid Precipitation Patterns and Trends in Eastern North America, 1980-84." J. Climate and Applied Met. $26: 980-994$.

Semonin, R. G. 1981. "Seasonal Precipitation Concentrations and Depositions for North America from the CANSAP/NADP Network." Nineteenth Progress Report to U.S. Department of Energy, Pollutant Characterization and Safety Division, Contract DE-ACO2-76EV01199.

Sweeney, J. K. and A. R. 01sen. 1987. Acid Precipitation in North America: 1985 Annual and Seasonal Data Summaries from Acid Deposition System Data Base. EPA/600/4-87/035, U.S. Environmental Protection Agency, Research Triangle Park, North Carolina.

Sweeney, J. K. and A. R. 01sen. 1989. Acid Precipitation in North America: 1986 Annual and Seasonal Data Summaries from Acid Deposition System Data Base. EPA/600/4-89/005, U.S. Environmental Protection Agency, Research Triangle Park, North Carolina.

Venkatram, A. 1988. "On the Use of Kriging in the Spatial Analysis of Acid Precipitation Data." Atmos. Environ. 22(9):1963-76. 
Vong, R., S. Cline, G. Reams, J. Bernert, D. Charles, J. Gibson, T. Haas, J. Moore, R. Husar, A. R. Olsen, J. C. Simpson, and S. Seilkop. 1989. Regional analysis of Wet Deposition for Effects Research. EPA/600/3-89/030, U.S. Environmental Protection Agency, Research Triangle Park, North Carolina.

Wampler, S. J. and A. R. 01sen. 1987. "Spatial Estimation of Annual Wet Acid Deposition Using Supplemental Precipitation Data." Tenth Conference on Probability and Statistics, pp. 248-251. American Meteorological Society, Boston.

Watson, C. R. and A. R. 01sen. 1984. Acid Deposition System (ADS) for Statistical Reporting. System Design and Users Code. EPA-600/8-84-023, U.S. Environmental Protection Agency, Research Triangle Park, North Carolina. 



\section{APPENDIX A}

COMPARISON OF $1985 \mathrm{pH}$ CONTOUR MAPS 
APPENDIX A

COMPARISON OF 1985 PH CONTOUR MAPS

Anthony R. 01sen

Pacific Northwest Laboratory

June 22, 1988

This paper gives a comparison of the $1985 \mathrm{pH}$ maps that appear in the NAPAP Interim Assessment (NAPAP 1987) and in the NADP 1985 Annual Data Summary report (NADP 1987).

Figure 1 reproduces the NADP report map and Figure 2 reproduces the NAPAP report contour map, prepared by Pacific Northwest Laboratory (PNL). The first feature of the maps that differs is the use of different contour levels. This makes any comparison difficult. Judgement of whether the maps agree depends on who makes the judgement and what is the intended purpose of the maps. An appropriate purpose for the map appears to be a semi-quantitative display of the spatial pattern of $\mathrm{pH}$ during 1985. With this as the purpose, the two maps appear to agree.

The production of contour maps is a problem of surface estimation and display. All solutions will not agree exactly in the location of contour lines but strong qualitative agreement should be expected. I have taken a closer look at the production process for the maps. First, I reviewed the production processes used by NADP and PNL to determine the steps used. Second, I requested that PNL and NADP prepare several alternative maps to investigate

possible reasons for quantitative differences in the maps.

\section{PRODUCTION PROCESSES}

The production of a contour map includes the following:

- Calculation of an annual pH value at a site,

- Selection of sites to be used in surface estimation,

- Selection of a surface estimate technique,

- Display of the estimated surface,

- Production of final document quality contour map.

NADP and PNL use the same calculation procedure to obtain an annual $\mathrm{pH}$ value for a site. The $\mathrm{PH}$ values for sites that NADP and PNL both used in preparing 
the maps agree in all cases. Both use $\mathrm{pH}$ to the nearest hundredth in the production process. Note that $\mathrm{pH}$ values that appear on the maps are rounded to the nearest tenth. Because of the rounding, contour lines may include or exclude sites that appear to be on the "wrong" side.

The NADP and PNL maps do use different sites for the surface estimation. NADP uses only NADP/NTN network sites. NADP used 123 NADP/NTN sites and PNL used 183 sites from multiple networks. PNL uses sites from the NADP/NTN, UAPSP, MAP3S, CAPMON, and APIOS networks. Fewer NADP sites (20) are used by PNL than by NADP. This is due to using different criteria for inclusion of a site. The major difference is that PNL includes a quarterly criteria as well as an annual criteria. The underlying data completeness measures are calculated the same, the difference is in which measures are applied and what cutoff criteria is used. Based on our current knowledge on the relationship between the criteria and the "representativeness" of the annual summary, I do not believe that a case can be made for preferring either the NADP or the PNL criteria. Both are reasonable choices.

NADP and PNL use entirely different surface estimation algorithms. The initial step in both algorithms is to estimate the surface on a regular grid. The regular grid is then used to determine the location of the contour lines. NADP uses a grid on a transverse mercator projection at an unknown but reasonable grid density. PNL uses an $80 \mathrm{~km}$ grid on a Lambert conic projection that is near-distance preserving. NADP uses the SURFACE II graphics system (Sampson 1984). The gridding routine uses a constrained distance-squared weighting function applied to the eight nearest site locations (a maximum search radius is imposed). PNL uses the kriging algorithms in BLUEPACK software (Delfiner 1979). Kriging also is distance-weighted but the distance weights are derived from the variation observed in the site data. The $\mathrm{pH}$ weights are from a spherical semi-variogram. Eight sites are used with a restriction that each octant from the grid node contributes a site, if available within a maximum search radius. 
Smooth contour lines are interpolated from the regular grid. NADP uses the "CONT" function in Surface II, which uses a piecewise Bessel interpolation within a grid cell. PNL uses the DISSPLA graphics contouring function with a cubic spline interpolation, termed "spline under tension." I review the maps that are computer generated for consistency with the monitoring site data. This review typically includes removing contours in the West, deleting extensions of contours over the ocean, and subjectively smoothing contours, especially in data sparse regions, to remove non-data supported features.

\section{ALTERNATIVE MAP COMPARISON}

Since NADP and PNL used different sets of sites and different surface estimation and contouring algorithms, four alternative maps are produced for the four possible combinations. As a point of departure, Figures 1 and 2 are the original maps as they appeared in the NAPAP interim assessment and the NADP 1985 Annual Data Sumnary. The sites included in the NADP data set (123 sites) and the PNL data set (183 sites) are shown in Figures 3 and 4 , respectively.

For the comparison, PNL produced two maps (Figures 5 and 6 ) using our standard surface estimation and contouring procedure. Contour levels were changed to be the same as the NADP original map. The same kriging semivariogram is used for both maps (same as used in NAPAP map). The maps provide an assessment of differences that arise from using different subsets of sites. My assessment is that the maps agree very well east of the Mississippi River within the United States. The 4.7 and 4.9 contours have bends in the south that are not supported by site data. These would be subjectively smoothed to remove "artificial" features. The 5.1 contour extends into the West where contouring is questionable. The 4.5 contour differs in Canada and Maine due to the PNL data set including Canadian sites. The 4.3 contour differs in northern New York. Dther differences in the contours are small. In Figure 7, an expanded view of eastern North America for the original PNL NAPAP map is given for comparison. 
NADP used the NADP contouring algorithm to produce a map based on the NADP data set sites (Figure 8 ) and the PNL data set sites (Figure 9). The maps agree very well east of the Mississippi within the United States. The differences between them are similar to those present between the two PNL maps. The areas, west of Mississippi and Canada, where the density of sites differs between the two data sets show the greatest differences.

The NADP map (Figure 8) and the PNL map (Figure 5) using the 123 sites in the NADP data set are remarkably similar. The north, northeast and southwest portion of the NADP 4.3 contour extends farther than the PNL contour. The southern portion of the 4.7 NADP contour extends into Texas while the PNL contour does not. Larger differences occur for the 5.1 contour in the west, reflecting sparse data support in this region. The NADP map (Figure 9) and the PNL map (Figure 6) using the 183 sites in the PNL data set show differences remarkably similar to the previous comparison.

\section{SUMMARY}

My assessment of the comparison of the different data sets and the different "contouring" algorithms used by NADP and PNL is that they produce remarkably similar maps. Agreement is best where the density of sites is greatest and poorest where the density is lowest. Inclusion of Canadian sites does aid in completing contours in the northeast. This is to be expected. When the algorithms are compared on the same data set, the differences are no greater than differences observed when using same algorithm with different data sets. The computer drawn NADP maps appear to be smoother than the computer drawn PNL maps. This is related to the density of grids used and the selection of a smoothing parameter. 


\section{REFERENCES}

Delfiner, P., J.P. Delhome, J.P. Chiles, D. Renard, and F Irigoin. 1979. BLUEPACK 3-D. Centre De Geostatistique et De Morphologie, Fontainebleau, France.

National Acid Deposition Program. 1987. NADP/NTN Annual Data Summary. Precipitation Chemistry in the United States. 1985. Natural Resource Ecology Laboratory, Colorado State University, Fort Collins, co.

National Acid Precipitation Assessment Program. 1987. Interim Assessment: The Causes and Effects of Acidic Deposition. Vol. III Atmospheric Process. U.S. Government Printing Office, Washington, DC.

Sampson, Robert J. 1984. SURFACE II GRAPHICS SYSTEM. Kansas Geological Survey, Lawrence, Kansas. 


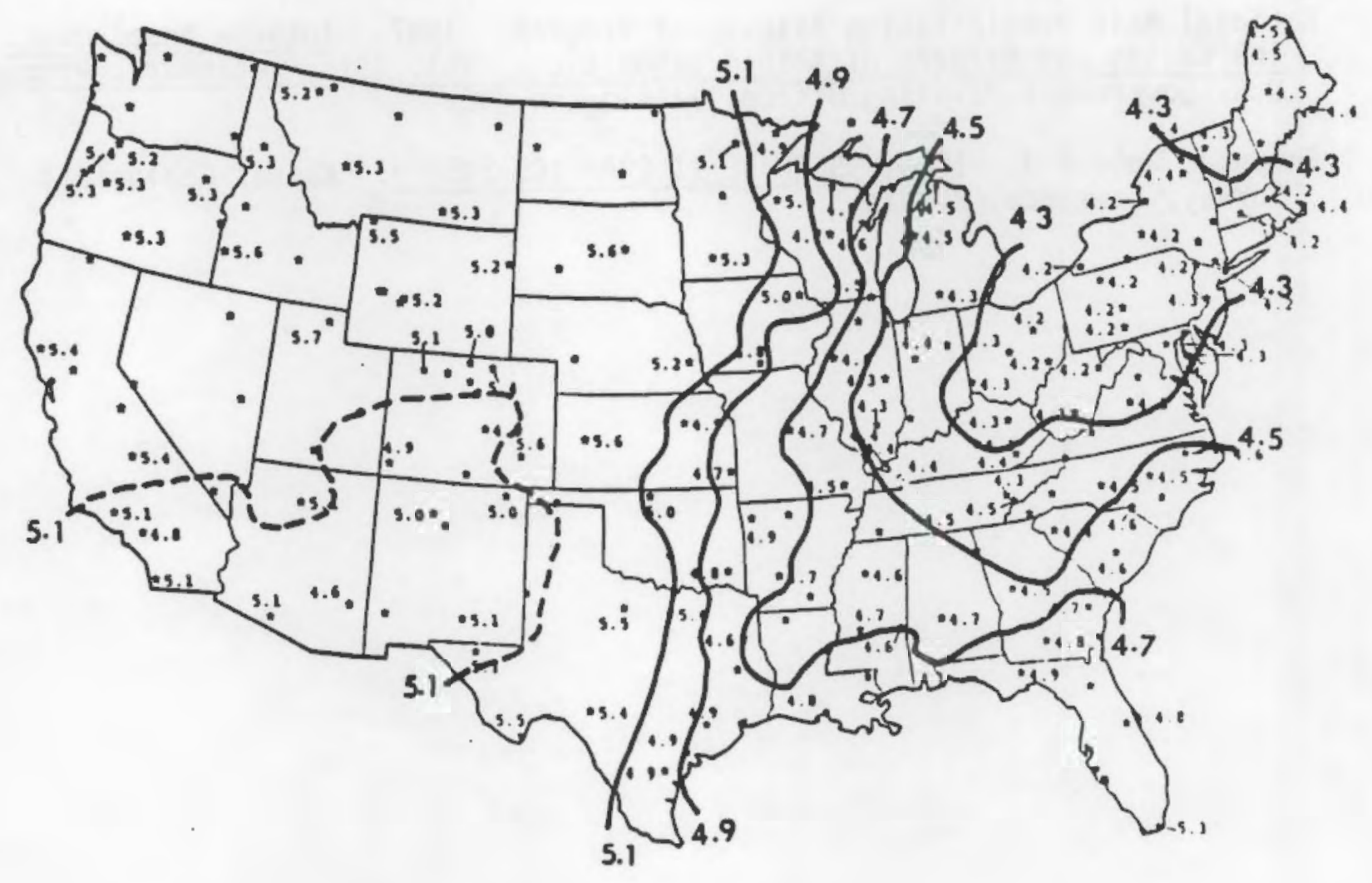

FIGURE 1. NADP Original 1985 Annual Data Summary pH Map 


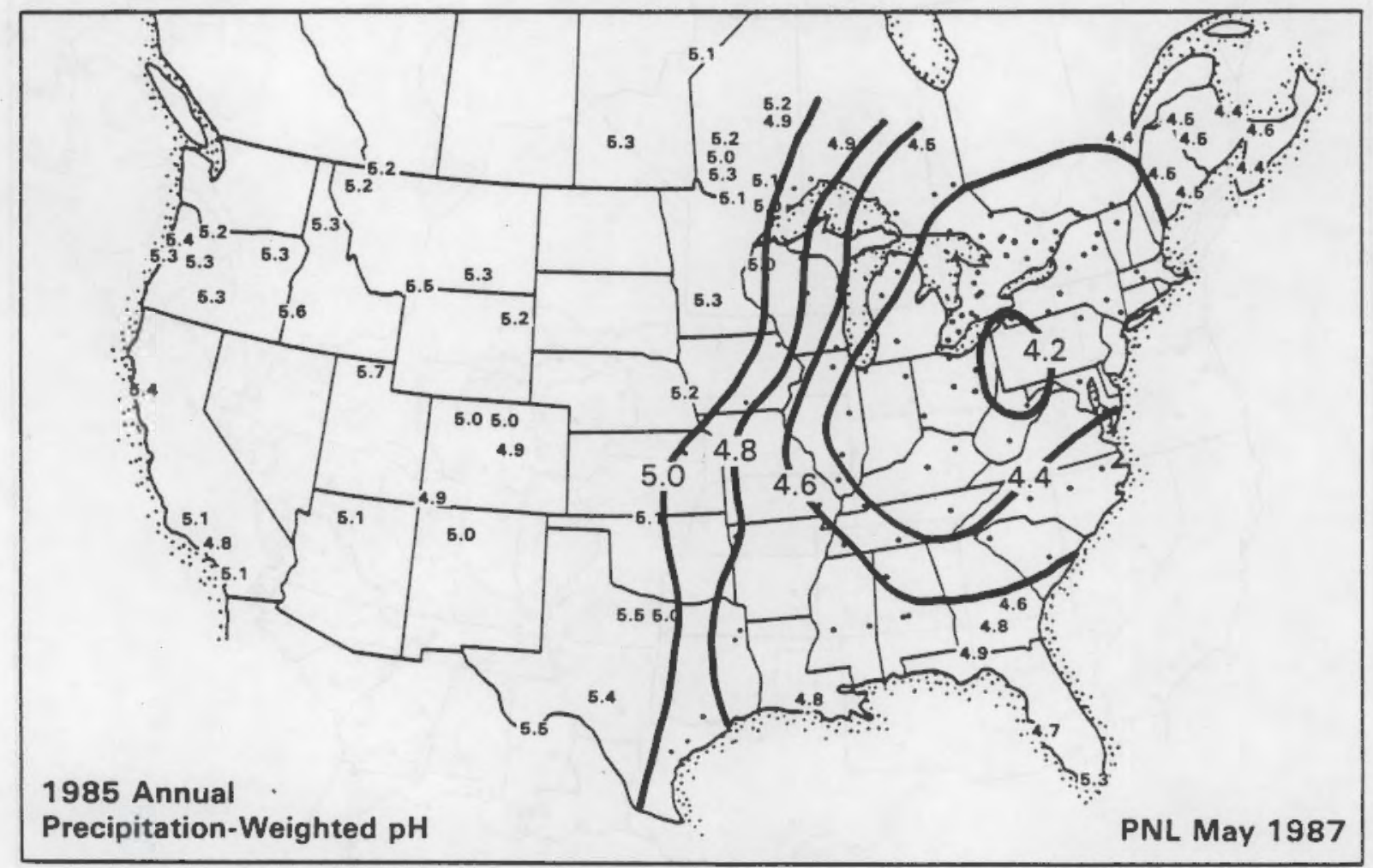

FIGURE 2. PNL Original NAPAP Interim Assessment Document 1985 pH Map 


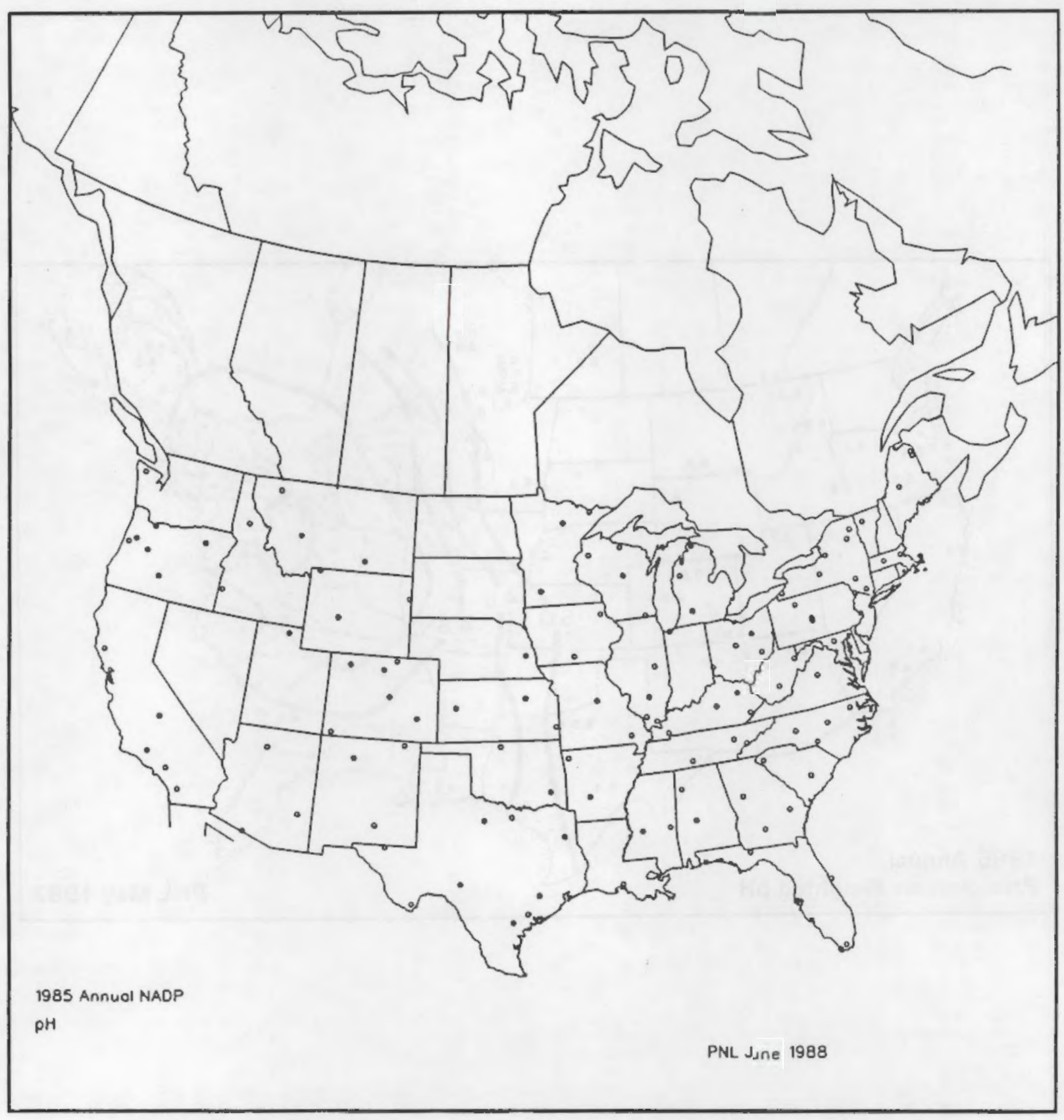

FIGURE 3. Sites Used in NADP pH Data Set 


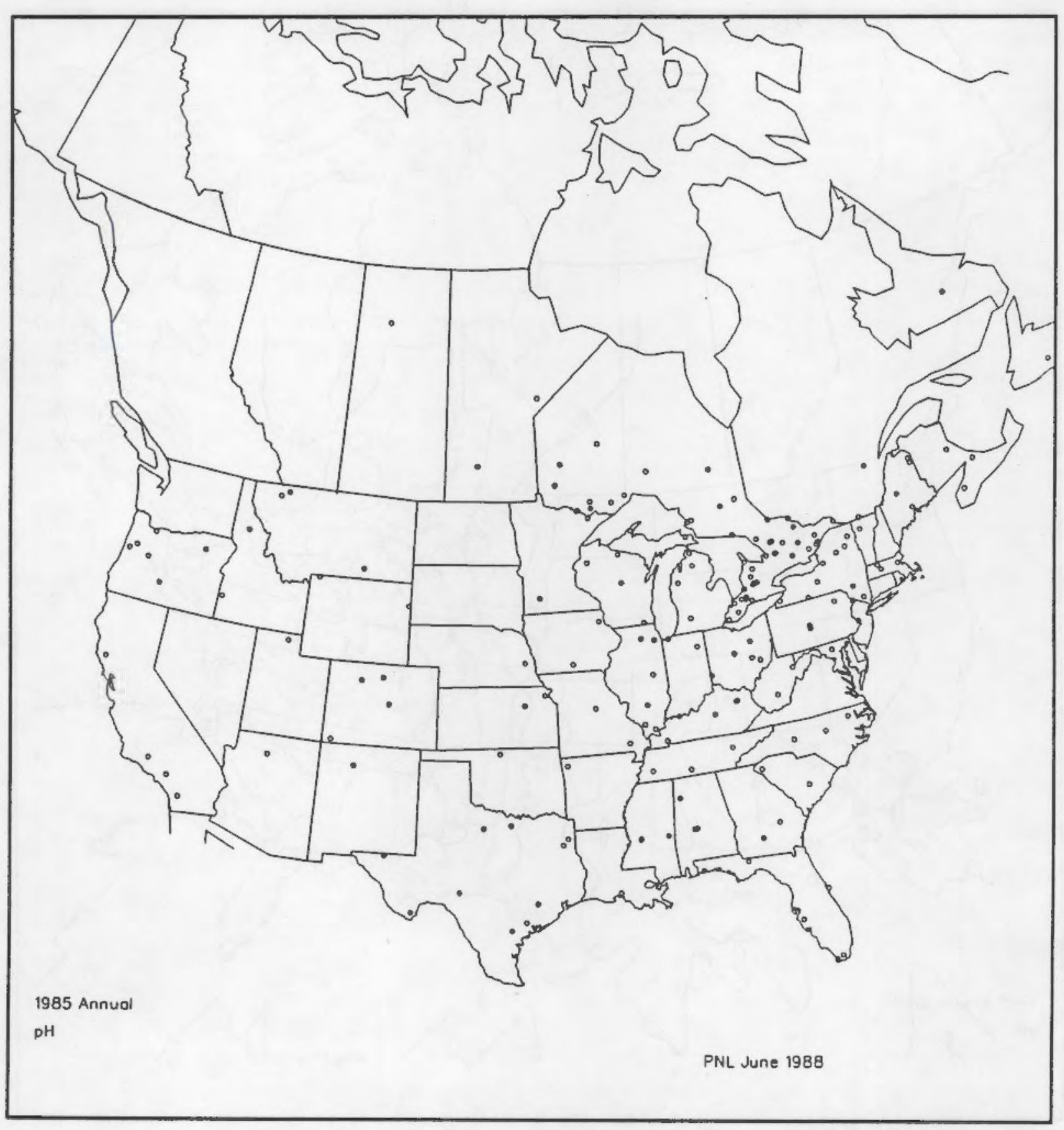

FIGURE 4. Sites Used in PNL pH Data Set 


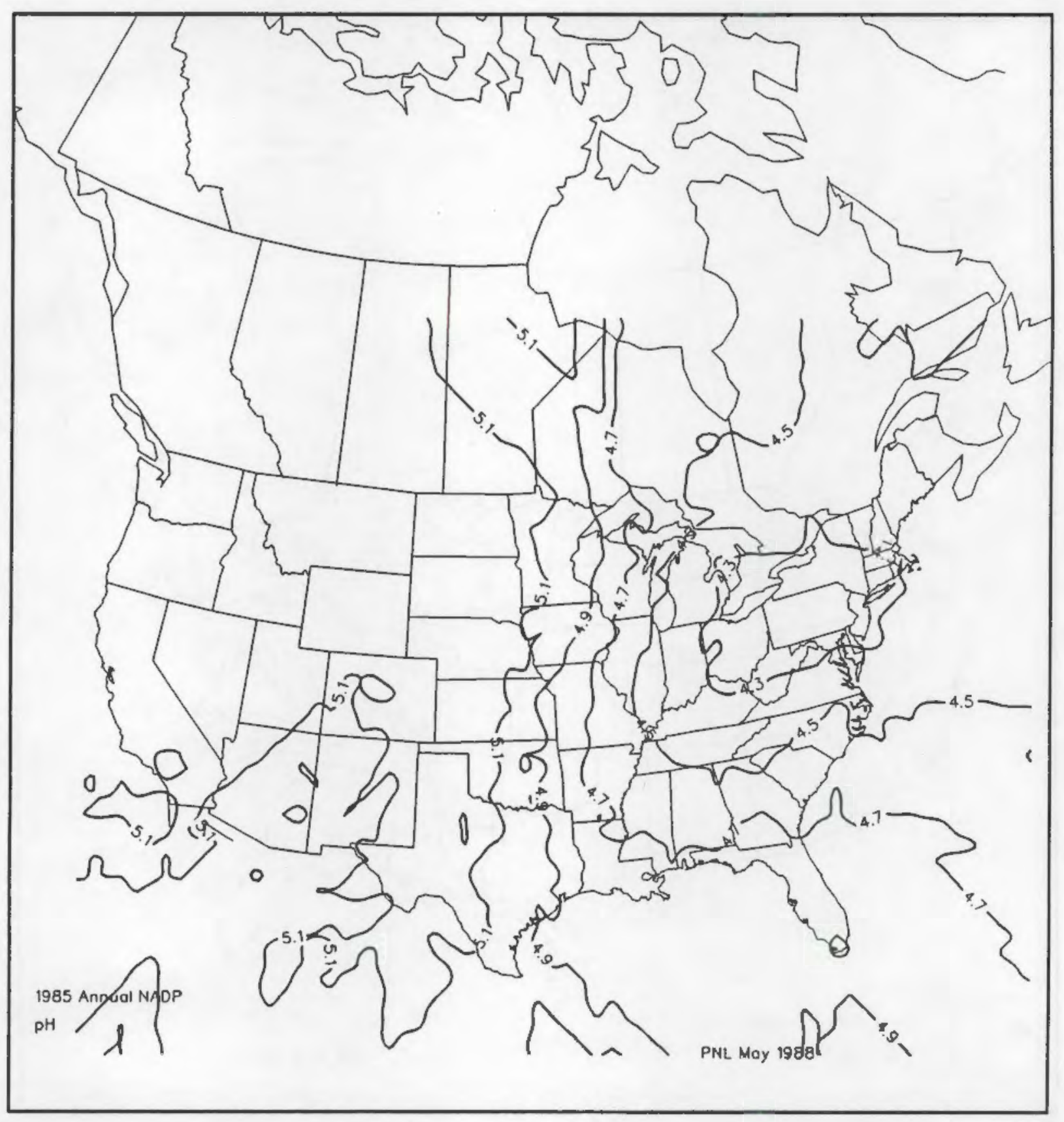

FIGURE 5. PNL Kriging Map Using NADP Data Set Sites 


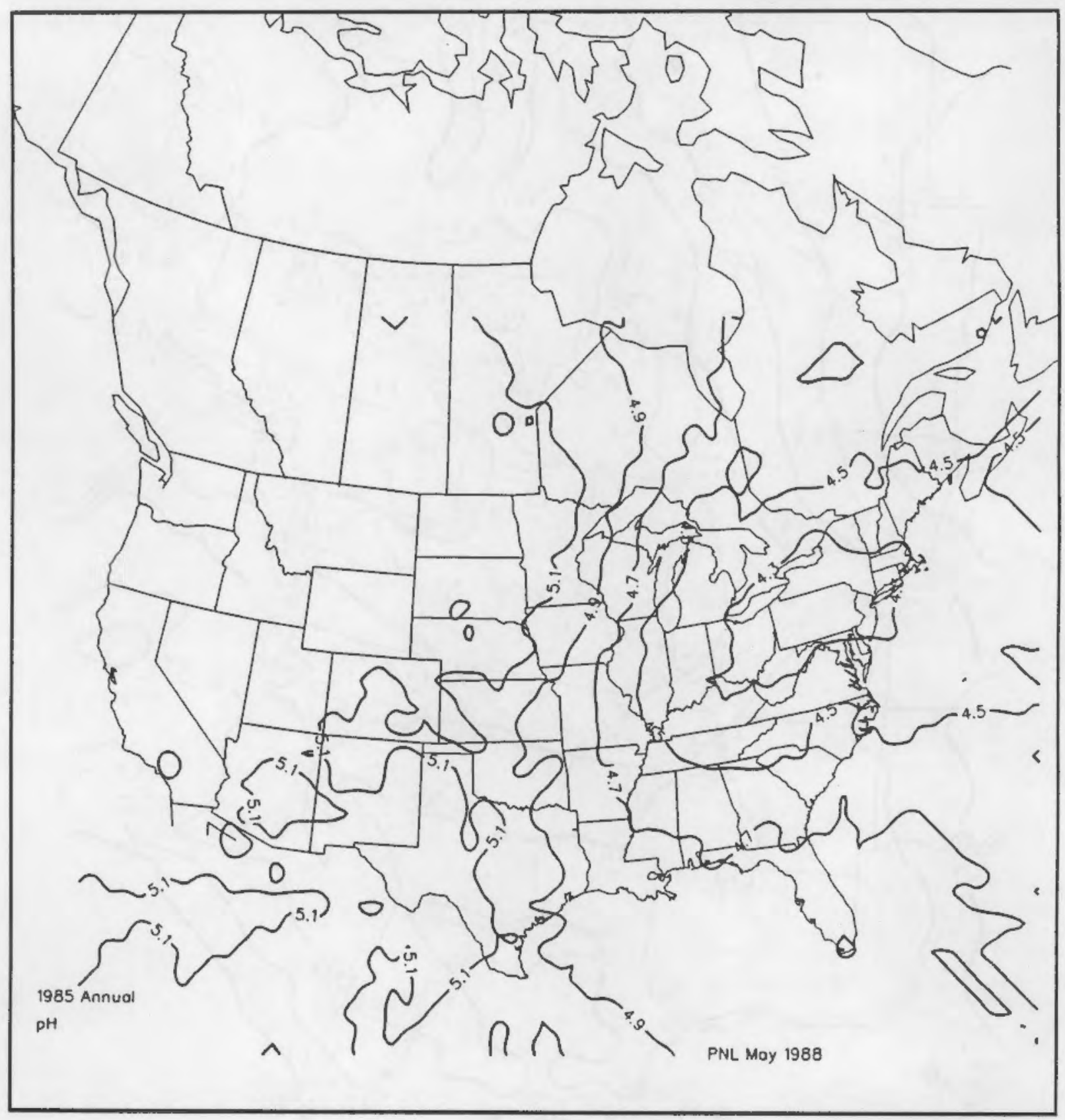

FIGURE 6. PNL Kriging Map Using PNL Data Set Sites 


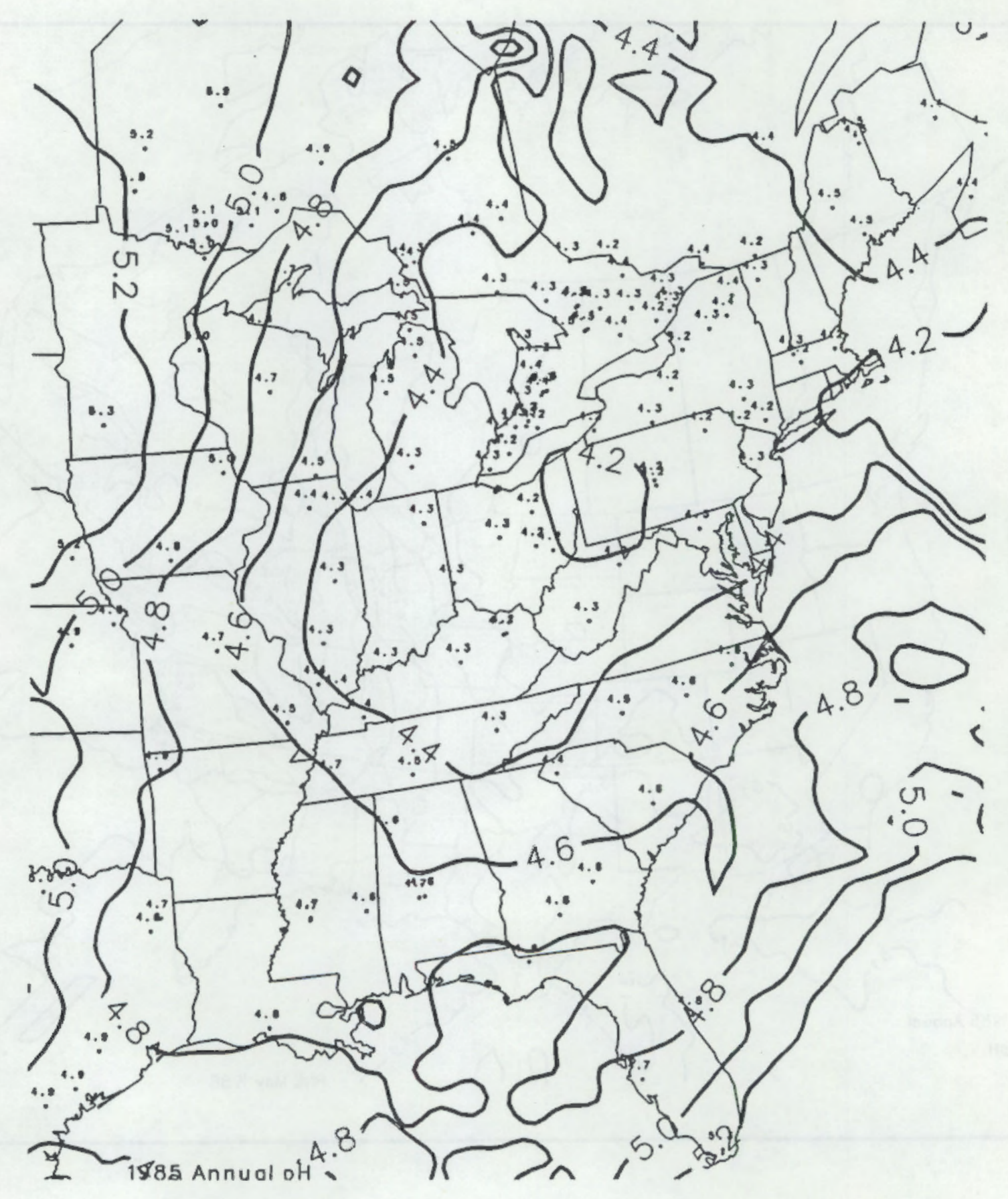

FIGURE 7. PNL Computer Drawn Map for Original NAPAP Interim Assessment pH Map 


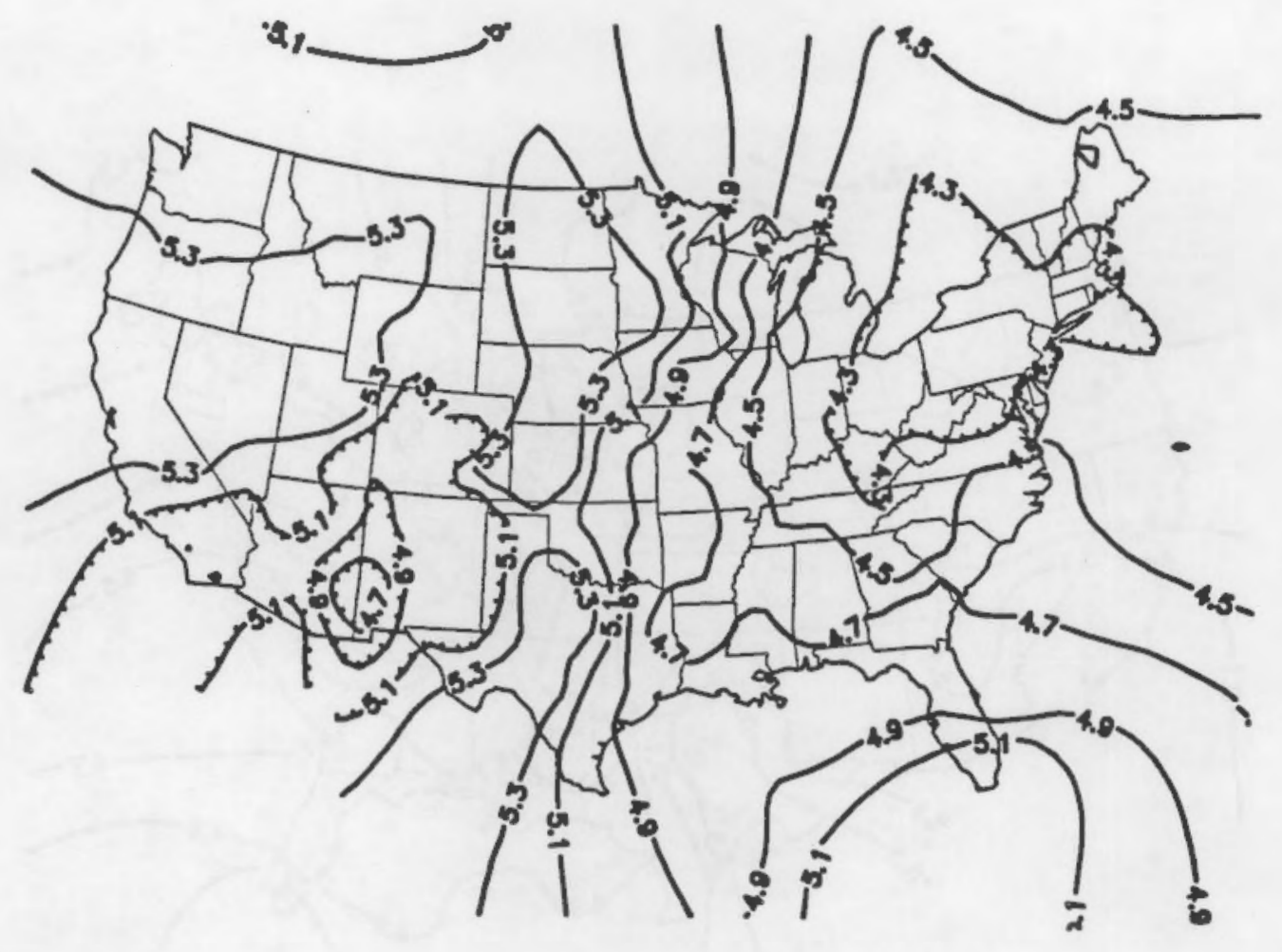

FIGURE 8. NADP Map Using NADP Data Set Sites 


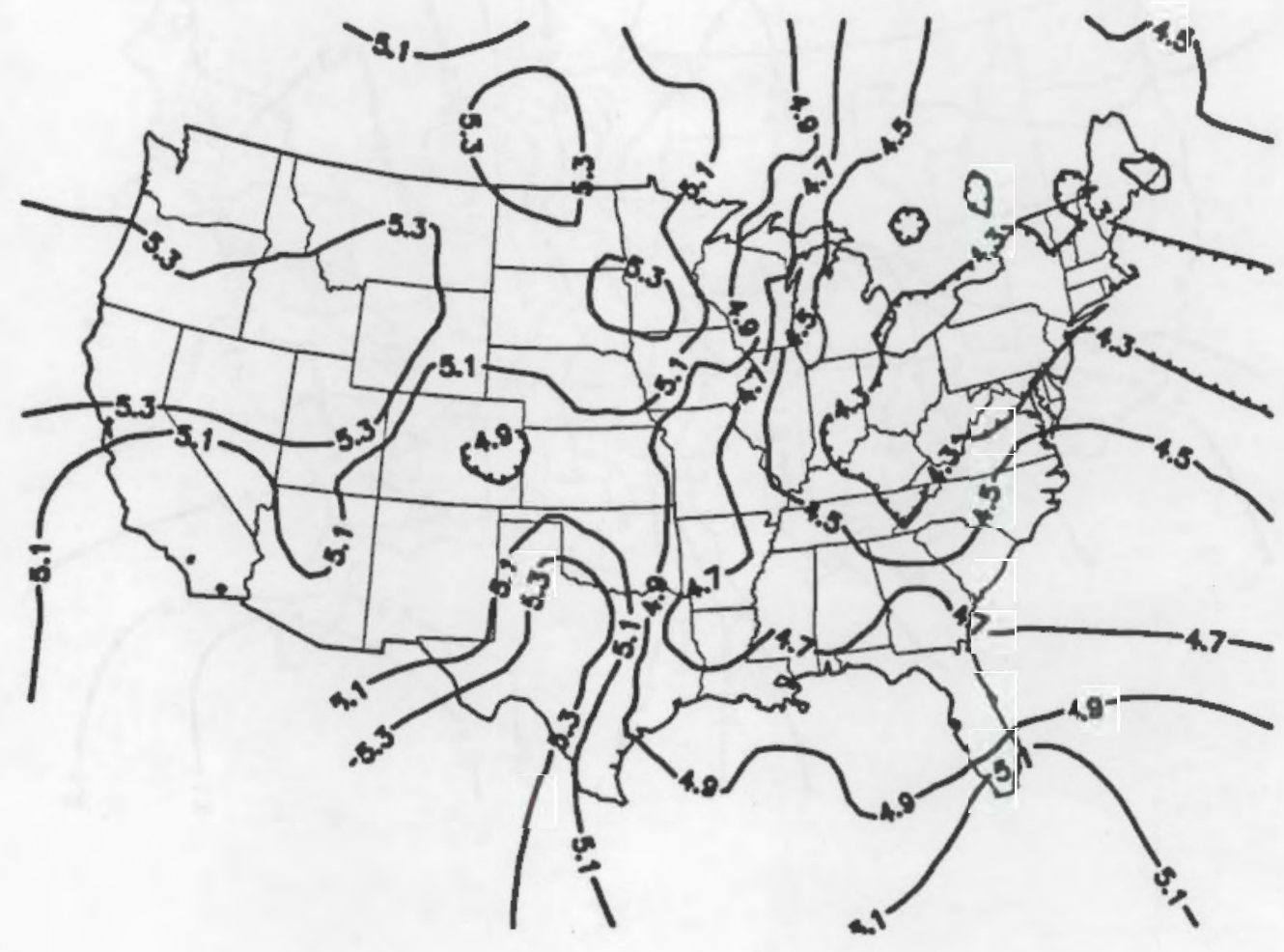

FIGURE 9. NADP Map Using PNL Data Set Sites 
PNL -7291

UC -402

\section{DISTRIBUTION}

No. of

Copies

OFFSITE

D. H. Holland

Design and Reports Branch MD-56

Atmospheric Research and Exposure Assessment Laboratory

U.S. Environmental Protection Agency

Research Triangle Park, NC 27711

12 DOE/Office of Scientific and

Technica1 Information

D. Beecy

U.S. Department of Energy

Office of Planning and

Environment

FE-13, Room C-176 (GTN)

Washington, DC 20545

G. Jordy

U.S. Department of Energy

Office of Program Analys is

MS ER-30, GTN

Washington, DC 20545

D. H. Slade

U.S. Department of Energy

ER-74, GTN

Hashington, DC 20545

D. Winstanley

U.S. Department of Energy

Office of Energy Research

$M S-G 226, E R-32$

Washington, DC 20545

M. A. Allan

Energy Analysis and Environment

Division

Electric Power Research Institute

P.0. Box 10412

Palo Alto, CA 94303
No. of

Copies

D. S. Bigelow

Natural Resource Ecology Laboratory

Colorado State University

Fort Collins, CO 80523

V. C. Bowersox

Central Analytical Laboratory

111 inois State Water Survey

2204 Griffith Drive

Champaign, IL 61820

S. Bromberg

Atmospheric Research and Exposure Assessment Laboratory, MD-75

U.S. Environmental Protection Agency

Research Triangle Park, NC 27711

R. Dennis

Atmospheric Research and Exposure Assessment Laboratory, MD-80

U.S. Environmental Protection Agency

Research Triangle Park, NC 27711

J. H. Gibson

Natural Research Ecology Laboratory

Colorado State University

Fort Collins, CO 80523

B. B. Hicks

NOAA - Air Resources Laboratory

Atmospheric Turbulence and

Diffusion Division

$456 \mathrm{~S}$. Illinois Avenue

Oak Ridge, TN 37830

F. P. Kapinos

U.S. Geological Survey

414 National Center

Reston, VA 22092 
No. of

Copies

\author{
W. W. Knapp \\ Department of Agronomy \\ 1111 Bradfield Hall \\ Cornell University \\ Ithaca, NY 14853 \\ J. Mahoney \\ Director of Research \\ NAPAP Office of the Director \\ 722 Jackson Place, NW \\ Washington, DC 20503
J. M. Miller
NOAA - Air Resources Laboratory
6010 Executive Boulevard
Rockville, MD 20852

V. A. Mohnen

Earth Sciences Bujlding, Room 324

State University of New York at Albany

1400 Washington Avenue

Albany, NY 12222

P. K. Muller

Environmental Assessment Department

Electric Power Research Institute P.0. Box 10412

Palo Alto, CA 94303

\author{
National Wildlife Federation \\ Toxics and Pollution Program \\ 1412 16th Street NW \\ Washington, DC 20036 \\ B. L. Niemann \\ Office of Air and \\ Radiation (OAR-455) \\ U.S. Environmental Protection \\ Agency \\ Washington, DC 20460
}

No. of

Copies

J. Pickering

U.S. Geological Survey, MS 416

412 National Center

Reston, VA 22092

D. Renne

NAPAP Office of the Director

722 Jackson Place, NW

Washington, DC 20503

F. Schiermeier

Atmospheric Research and Exposure Assessment Laboratory, MD-80

U.S. Environmental Protection Agency

Research Triangle Park, NC 27711

R. G. Semonin

Illinois State Water Survey

2204 Griffith Drive

Champaign, IL 61820

D. She iman

Natural Resources Defense Council

1350 New York, NW

Washington, DC 20005

D. Sisterson

Argonne National Laboratory

9700 South Cass Avenue

Argonne, IL 60439

G. J. Stens 1 and

Illinois State Water Survey

2204 Griffith Drive

Champaign, IL 61820

R. J. Vong

U.S. Environmental Protection Agency

200 SW 35th Street

Corvallis, OR 97333 
No. of

Copies

D. Winters

Office of Air and

Radiation (OAR-455)

U.S. Environmental Protection Agency

Washington, DC 20460

\section{FOREIGN}

L. A. Barrie

Atmospheric Environment Service 4905 Dufferin Street

Downsview, Ontario M3H 5T4

CANADA

H. Dovland

Norwegian Institute for Air Research

P.B. 130, N-2001 Lillestrom

Elvegt. 52

NORWAY

\section{Granat}

Department of Meteorology

University of Stockholm

Arrhenius Laboratory

S-106 91 Stockholm

SWEOEN

M. A. Lus is

Air Resources Branch

Ontario Ministry of the Environment

880 Bay Street

Toronto, Ontario M5S IZ8

CANADA

J. Schaug

Norwegian Institute for Air Research

P.B. 130, N-2001 Lillestrom Elvegt. 52

NORWAY
No. of

Copies

\author{
P. W. Summers \\ Atmospheric Environment Service \\ 4905 Dufferin Street \\ Downsview, Ontario M3H 5T4 \\ CANADA
}

R. J. Vet

Atmospheric Environment Service 4905 Dufferin Street

Downsview, Ontario M3H 5T4

CANADA

E. Voldner

Atmospheric Environment Service 4905 Dufferin Street

Downsview, Ontario M3H 5T4

CANADA

D. M. Whelpdale

Atmospheric Environment Service 4905 Dufferin Street

Downsview, Ontario M3H 5T4

CANADA

J. V. Zidek

University of British Columbia

2021 West Mall

Vancouver, BC V6T 1W5

CANADA

\section{ONSITE}

26 Pacific Northwest Laboratory

W. R. Barchet

R. L. Cheatham

M. T. Dana

J. M. Hales

A. R. 01sen (15)

J. C. Simpson

Publishing Coordination

Technical Report Files (5) 
